IFIC/02-36,FTUV-03-0212

$\mathrm{IPPP} / 02 / 49, \mathrm{DCPT} / 02 / 98$

\title{
Present and Future Bounds on Non-Standard Neutrino Interactions
}

\author{
S. Davidson ${ }^{a}$, C. Peña-Garay ${ }^{b}$, N. Rius ${ }^{c}$, A. Santamaria ${ }^{c}$ \\ (a) IPPP, University of Durham, Durham DH1 3LE,UK \\ (b) School of Natural Sciences, Institute for Advanced Study, Princeton, NJ 08540 \\ (c) Depto. de Física Teórica and IFIC, Universidad de Valencia-CSIC, Edificio de Institutos de Paterna, Apt. \\ 22085, 46071 Valencia, Spain
}

\begin{abstract}
We consider Non-Standard neutrino Interactions (NSI), described by four-fermion operators of the form $\left(\bar{\nu}_{\alpha} \gamma \nu_{\beta}\right)(\bar{f} \gamma f)$, where $f$ is an electron or first generation quark. We assume these operators are generated at dimension $\geq 8$, so the related vertices involving charged leptons, obtained by an $S U(2)$ transformation $\nu_{\delta} \rightarrow e_{\delta}$, do not appear at tree level. These related vertices necessarily arise at one loop, via $W$ exchange. We catalogue current constraints from $\sin ^{2} \theta_{W}$ measurements in neutrino scattering, from atmospheric neutrino observations, from LEP, and from bounds on the related charged lepton operators. We estimate future bounds from comparing KamLAND and solar neutrino data, and from measuring $\sin ^{2} \theta_{W}$ at the near detector of a neutrino factory. Operators constructed with $\nu_{\mu}$ and $\nu_{e}$ should not confuse the determination of oscillation parameters at a $\nu$ factory, because the processes we consider are more sensitive than oscillations at the far detector. For operators involving $\nu_{\tau}$, we estimate similar sensitivities at the near and far detector.
\end{abstract}

\section{Introduction}

Lepton flavour violation is observed in atmospheric and solar neutrino experiments. The atmospheric neutrino deficit is mainly due to muon neutrino disappearance: the $\nu_{\mu}$ flux measured at Super-Kamiokande (SK) has a strong zenith angle dependence, which deviates from the Standard Model (SM) expectation by more than $7 \sigma$ [1]. Neutrinos change flavors as they travel to the Earth from the center of the Sun, as was seen directly at the Sudbury Neutrino Observatory (SNO) through the measurements of the charged current and the neutral current reactions for ${ }^{8} B$ solar neutrinos [2].

These results demonstrate simply that new physics is required. In principle, the atmospheric and solar neutrino deficits can be explained by neutrino masses or by giving the neutrinos new interactions. Data disfavor non standard interactions ( NSI) of neutrinos as an explanation for the atmospheric neutrino deficit $[3,4,5]$ through the energy and baseline dependence. KamLAND detector has recently confirmed the large mixing angle (LMA) oscillation explanation of the solar neutrino puzzle[6]. Prior to this, NSI were a viable alternative solution[7, 8] *

Neutrino masses are the leading mechanism in the solar and atmospheric anomalies. Nonetheless, NSI may be comparable to (or larger than) oscillation effects in other processes or at other energies. This is a particularly relevant issue for neutrino factories $[10,11,12,13,14,15,16,17]$, where NSI may affect the oscillation parameters inferred from experimental data, biasing the value of some of the mixing angles. For instance, it was suggested in [16] that NSI

\footnotetext{
*Reference [9] found that NSI induced in the R-parity violating MSSM could not explain the solar neutrino deficit. However, their operators were otherwise constrained to be at least an order of magnitude smaller than the solutions found in [8].
} 
could interfere with the determination of $\sin ^{2} \theta_{13}$ at a neutrino factory ${ }^{\dagger}$. It is important to understand how significant is this possibility. The original aim of this paper was to show that short baseline and precision experiments are more sensitive to NSI than the far detector at a neutrino factory. We will see that this is true for some NSI, but borderline for those involving a $\nu_{\tau}$.

We consider neutral current NSI, from a phenomenological perspective: we follow [18], and assume that the new physics which induces the non-standard $(\bar{\nu} \nu)(\bar{f} f)$ operator, where $f$ is a charged lepton $(\ell)$ or quark, does not introduce new charged lepton physics at tree level. Within this approach, NSI can be constrained $[18,19]$ from neutrino deep inelastic scattering experiments and from elastic scattering $\nu-e$, where the baseline is too short for oscillations. NSI would contribute to $\nu$ scattering events, and therefore to the determination of $\sin ^{2} \theta_{W}$ in these experiments. So short baseline, high-flux neutrino experiments, that measure for instance $\sin ^{2} \theta_{W}$, can set significant bounds on NSI involving $\nu_{e}$ and $\nu_{\mu}$.

A $(\bar{\nu} \nu)(\bar{f} f)$ operator, will nonetheless induce a $(\bar{\ell} \ell)(\bar{f} f)$ and/or $(\bar{\ell} \nu)\left(\bar{f} f^{\prime}\right)$ operator, where $f^{\prime}$ is the $\mathrm{SU}(2)$ partner of $f$, via external one-loop Standard Model dressing. This is independent of the new physics that induces the $(\bar{\nu} \nu)(\bar{f} f)$ operator. Even in the case in which there are no tree level bounds on NSI of neutrinos, because there is no appropriate experiment, in general radiative corrections will generate other types of interactions which could be tested at present (or future) experiments. Bounds on charged lepton operators therefore set model independent bounds on the NSI operators. However, at present these bounds are only significant for $\mu-e$ flavor changing operators, because the loop suppression is a small number. NSI in loops can also make flavour dependent contributions to the decay rates of the electroweak gauge bosons, which can set relevant bounds on flavour diagonal NSI.

Present bounds still allow sizable (diagonal) NSI of $\nu_{\tau}$, but we will show that they can be constrained by comparing SNO/SK solar data and KamLAND results. The bounds are significantly better if KamLAND finds an oscillatory signal, that is if $\Delta m_{\text {sol }}^{2} \lesssim 10^{-4} \mathrm{eV}$, as we will assume for definiteness.

We introduce our notation and assumptions in section 2. Section 3 presents current constraints on flavour diagonal and flavour changing neutral current NSI, both from tree level effects (short baseline, high-flux neutrino scattering experiments, LEP, atmospheric neutrinos) and from one-loop processes (flavour changing charged lepton interactions, LEP). In section 4, we discuss the sensitivity of solar neutrino experiments, SNO and Super-Kamiokande, using KamLAND to pin-point the solar oscillation parameters, $\Delta m_{\text {sol }}^{2}$ and $\theta_{12}$. We also discuss the sensitivity of future experiments, using the near detector at a nufactory as an example. We summarize our results in section 6 , tabulating the best current and future bounds we obtained.

\section{Notation and Assumptions}

At energy scales $\ll m_{W}$ (where there is a large amount of precise $\nu$ scattering data), the Standard Model interactions of neutrinos can be described by the effective Lagrangian

$$
\mathcal{L}_{e f f}=-2 \sqrt{2} G_{F}\left(\left[\bar{\nu}_{\beta} \gamma_{\rho} L \ell_{\beta}\right]\left[\bar{f} \gamma^{\rho} L f^{\prime}\right]+\text { h.c. }\right)-2 \sqrt{2} G_{F} \sum_{P, f, \beta} g_{P}^{f}\left[\bar{\nu}_{\beta} \gamma_{\rho} L \nu_{\beta}\right]\left[\bar{f} \gamma^{\rho} P f\right]
$$

where $P=\{L, R\}, \ell$ is a charged lepton, $f$ is a lepton or quark, $f^{\prime}$ its $\mathrm{SU}(2)$ partner, and the $Z$ couplings $g_{P}^{f}$ are given in table 1. Greek indices from the beginning of the alphabet $(\alpha, \beta, \ldots)$ label lepton flavours, roman indices $(i, j, \ldots)$ correspond to neutrino mass eigenstates, and late alphabet Greek letters $(\rho, \sigma \ldots)$ are space-time indices.

We consider non-standard, neutral current neutrino interactions, so we add operators with the form of the second term in equation (1). We do not include new charged current interactions. As discussed in [10, 12, 14, 16, 20], NSI can contribute to a "neutrino oscillation" signal via charged current interactions in the source or detector, or via neutral current interactions in the propagation from source to detector. However, we anticipate that other experimental processes are more sensitive to charged current NSI that long baseline neutrino oscillations (e.g. flavour changing NSI in the source could induce taus and wrong sign muons in the near detector).

Non-renormalisable operators involving a Standard Model neutrino and anti-neutrino can be ordered by their dimension, or by their number of legs in the $S U(3) \times U(1)$ invariant effective theory of SM fermions and photons. These options are different, because Higgs fields saturated by the vacuum expectation value (vev) are not counted as legs. If we count by legs, then new physics coupled to neutrinos can appear as a four fermion operator. We require this operator to conserve electric charge, colour and lepton number, which forces it to be of $V \pm A$ form. We assume three light neutrinos, with Majorana masses and the SM interactions of equation (1). We allow them to have NSI, parametrised as

$$
\mathcal{L}_{e f f}^{N S I}=-\varepsilon_{\alpha \beta}^{f P} 2 \sqrt{2} G_{F}\left(\bar{\nu}_{\alpha} \gamma_{\rho} L \nu_{\beta}\right)\left(\bar{f} \gamma^{\rho} P f\right)
$$

${ }^{\dagger}$ This interference might be solved through the tail of the spectra at large energies (Ref. [17]). 


\begin{tabular}{|c|cc|}
\hline$Z$ couplings & $g_{L}^{f}$ & $g_{R}^{f}$ \\
\hline$\nu_{e}, \nu_{\mu}, \nu_{\tau}$ & $\frac{1}{2}$ & 0 \\
$e, \mu, \tau$ & $-\frac{1}{2}+\sin ^{2} \theta_{W}$ & $\sin ^{2} \theta_{W}$ \\
$u, c, t$ & $\frac{1}{2}-\frac{2}{3} \sin ^{2} \theta_{W}$ & $-\frac{2}{3} \sin ^{2} \theta_{W}$ \\
$d, s, b$ & $-\frac{1}{2}+\frac{1}{3} \sin ^{2} \theta_{W}$ & $\frac{1}{3} \sin ^{2} \theta_{W}$ \\
\hline
\end{tabular}

Table 1: $Z$ couplings to SM fermions.

where $f$ is now a first generation SM fermion: $e, u$ or $d$, and $P=L$ or $R$. We are not concerned with $f$ from the second or third generation, because such interactions could not affect oscillation experiments. We neglect possible CP violation in the new interactions (this has been considered in $[13,21]$ ), so we take $\varepsilon_{\alpha \beta}^{f P} \in \Re$. If the only new neutrino interactions are neutral current, the neutrino flavour basis is well-defined (see [20]), and we can label neutrinos by their SM flavour.

Assuming that the four-fermion vertices of equation (2) arise in an $S U(2) \otimes U(1)$ gauge invariant theory containing the SM spectrum with a single Higgs doublet, they can be generated by operators of dimension six, eight and larger [18] containing more and more vevs of the Higgs doublet. In this paper we will not consider dimension seven $\Delta L=2$ operators [22] because, unless their coupling is very small, we expect these interactions to generate too large neutrino Majorana masses. There is an important difference between the dimension 6 and dimension $8(\bar{\nu} \nu)(\bar{f} f)$ operators: new physics which induces the dimension 6 operator also induces an operator involving charged leptons, with a coefficient of the same order (by $S U(2)$ invariance) [23]. Charged lepton physics imposes tight constraints on these coefficients of dimension 6 operators. At dimension 8, an operator as in equation (2) can appear at tree level, for instance as

$$
\bar{e}_{R}\left(H^{\dagger} \sigma^{a} \ell\right)\left(\bar{\ell} \sigma^{a} H\right) e_{R} \rightarrow-\frac{1}{2}\langle H\rangle^{2}\left(\bar{e} \gamma^{\rho} R e\right)\left(\bar{\nu} \gamma_{\rho} L \nu\right)
$$

without any charged lepton counterpart $[18]^{\ddagger}$. We include the operator (2), and neglect the associated tree-level charged current vertex, so we are effectively assuming that these operators are generated at dimension eight.

If new physics operators are generated at tree level by exchange of particles with mass $\Lambda$, naive power counting tells us that dimension six operators should give rise to $\varepsilon \sim h^{2} v_{F}^{2} / \Lambda^{2}$ where $h$ is some generic coupling of the new physics particles. Taking into account present data collected at LEP and Tevatron one can reasonably assume that $\Lambda>200 \mathrm{GeV}$. Even though somehow lighter particles are not excluded (e.g. if neutral or only produced in pairs or having small couplings with ordinary particles), we are using an effective Lagrangian approach which is only valid below $\Lambda$. This counting tells us that we expect $\varepsilon<h^{2}$ for dimension 6 operators. If NSI interactions are generated by dimension 8 operators one expects an extra suppression proportional to $v_{F}^{2} / \Lambda^{2}$ which is only important if $\Lambda \gg v_{F}$, therefore one naturally expects $\varepsilon \ll 1$. However, $h$ could be relatively large without leaving the perturbative regime. Thus, $\varepsilon$ 's order one are not completely unnatural if the scale of new physics is not extremely large and the couplings of new particles are large. It is, therefore, important to check how large these NSI can be on purely phenomenological grounds. Thus, in this paper, we will concentrate on the phenomenology of $U(1) \times S U(3)$ invariant $\left(\bar{\nu}_{\alpha} \nu_{\beta}\right)(\bar{f} f)$ operators, with arbitrary coefficients. As mentioned in the introduction, there will nonetheless be constraints from charged lepton physics on these operators. One loop SM dressing of the tree level NSI vertices $\left(\bar{\nu}_{\alpha} \nu_{\beta}\right)(\bar{f} f)$ (for instance exchanging a $W$ between the external legs), will necessarily induce $\left(\bar{e}_{\alpha} e_{\beta}\right)(\bar{f} f)$ and/or $\left(\bar{\nu}_{\alpha} e_{\beta}\right)\left(\bar{f} f^{\prime}\right)$. This is discussed in section 3.2 .

In the effective theory described by equations (1) and (2), there will normally be a number of four-fermion operators which can contribute to a process. Experimental measurements of different processes will set limits on different sums of operators. It is common, in setting a limit on a given four-fermion operator, to assume that all other four-fermion operators are zero. This approach makes sense when the new physics contributions add incoherently,c'est à dire when one adds probabilities not amplitudes. It also makes sense if the different new physics amplitudes interfere with different SM amplitudes - for instance, if there is an NSI - SM interference term $\propto \varepsilon_{\alpha \alpha}^{q L} g_{L}^{q}+\varepsilon_{\alpha \alpha}^{q R} g_{R}^{q}$, these two terms can only cancel against each other if the NSI know $\sin ^{2} \theta_{W}$. We consider this unlikely (since we assume they are generated at dimension 8 , rather than 6 ), so we quote limits on one $\varepsilon$ at a time in our summary tables.

We will assume that the only unknown new physics are the non-standard neutrino interactions. We calculate what current experiments should measure according to the SM, and require the NSI contribution to be less than the experimental error $(1.6 \sigma)$, or less than the theory - experiment discrepancy.

\footnotetext{
${ }^{\ddagger}$ The Lorentz indices in eq. (3) are contracted $\bar{\ell} e_{R}$. The $\sigma$ s carry gauge $S U(2)$ indices.
} 


\section{Present bounds}

Before considering particle physics bounds, let us discuss briefly astrophysical and cosmological bounds on neutrino NSI. Neutrino interactions with matter, electrons and light quarks, can affect many astrophysical and cosmological scenarios. They could keep neutrinos in thermal equilibrium with ordinary matter for a longer time at the time of nucleosynthesis and disturb one of the great successes of modern cosmology. They could also produce stronger interactions of neutrinos with matter in the core of supernovae therefore keeping neutrinos trapped for a longer time and disturbing the duration of the neutrino pulse observed in SN1987A [24, 25, 26]. They could also contribute to the energy loss of stars due to processes like plasmon decay $\left(\gamma^{*} \rightarrow \nu \bar{\nu}\right)$ which are determinant for the evolution of red giants. All these processes occur in the SM mediated by neutral or charged currents and the SM value is essential to understand the three scenarios mentioned. Now the question is how large NSI are allowed in order not to disturb present observations? For instance, the case of plasmon decay in red giants has been used to place stringent bounds on a possible neutrino magnetic moment[24]. The SM gives contributions to plasmon decay from neutral and charged currents for $\nu_{e}$ and only from neutral currents for $\nu_{\mu}$ and $\nu_{\tau}$. To destabilize the SM results one should modify some of these interactions by a factor larger than one[24]. Therefore, generically one can say that $\varepsilon<\mathcal{O}(1)$ from energy loss in red giants. We expect, at best, similar results from supernova data or nucleosynthesis. As we will see, laboratory data already place better limits on $\nu_{e}$ and $\nu_{\mu}$ NSI, and SNO, Super-Kamiokande and KamLAND will also improve the bounds for $\nu_{\tau}$.

We first consider tree level effects of the operators in (2), which contain only the neutrino current with either the electron or first generation quark currents. Low energy scattering experiments can constrain NSI involving $\nu_{e}$ and $\nu_{\mu}$, while to derive bounds on diagonal $\nu_{\tau}$ NSI one should use the measurement of the $e^{+} e^{-} \rightarrow \nu \bar{\nu} \gamma$ cross section at LEP [18] and atmospheric neutrino data [5]. We set further bounds using the fact that such operators always induce one loop effects in much better tested charged lepton processes. These constrain flavor changing NSI involving first and second generation neutrinos to be undetectably small.

\subsection{Tree level effects}

\subsection{1 $\nu$ scattering experiments}

Neutrino NSI with either electrons or first generation quarks can be constrained by low energy scattering data. We review previous analysis $[19,18]$ and update them by including the recent results of the NuTeV experiment. As we shall see below, the bounds are rather stringent for $\nu_{\mu}$ interactions, looser for $\nu_{e}$ and do not exist for (diagonal) $\nu_{\tau}$.

We present bounds assuming that only one operator is present at a time, for the reasons explained above, although we also comment on how these limits are relaxed when several diagonal NSI are considered simultaneously.

- $\nu_{e} e \rightarrow \nu e$ scattering

In the presence of neutral current neutrino NSI the $\nu_{e} e$ elastic cross section is given by

$$
\sigma\left(\nu_{e} e \rightarrow \nu e\right)=\frac{2 G_{F}^{2} m_{e} E_{\nu}}{\pi}\left[\left(1+g_{L}^{e}+\varepsilon_{e e}^{e L}\right)^{2}+\sum_{\alpha \neq e}\left|\varepsilon_{\alpha e}^{e L}\right|^{2}+\frac{1}{3}\left(g_{R}^{e}+\varepsilon_{e e}^{e R}\right)^{2}+\frac{1}{3} \sum_{\alpha \neq e}\left|\varepsilon_{\alpha e}^{e R}\right|^{2}\right]
$$

where $g_{L}^{e}=-0.2718$ and $g_{R}^{e}=0.2326$ are the SM neutral current couplings of the electron, including electroweak radiative corrections and corresponding to the best fit point of the latest SM global fit of precision observables (without including $\mathrm{NuTeV}$ ).

The most accurate measurement of this cross section is the LSND result [27]:

$$
\sigma\left(\nu_{e} e \rightarrow \nu e\right)=(1.17 \pm 0.17) \frac{G_{F}^{2} m_{e} E_{\nu}}{\pi},
$$

which, taking into account the SM prediction $\left.\sigma\left(\nu_{e} e \rightarrow \nu_{e} e\right)\right|_{S M}=1.0967 G_{F}^{2} m_{e} E_{\nu} / \pi$, translates into the following $90 \%$ CL bounds on diagonal $\nu_{e} e$ NSI (assuming only one operator at a time):

$$
\begin{array}{r}
-0.07<\varepsilon_{e e}^{e L}<0.11 \\
-1 .<\varepsilon_{e e}^{e R}<0.5
\end{array}
$$

We can also set bounds on flavour changing NSI. These are only relevant for $\nu_{\tau} \nu_{e}$ interactions, because for $\nu_{\mu} \nu_{e}$ better bounds are obtained from the one loop effects discussed in section 3.2. Assuming there are only flavor changing NSI we obtain:

$$
\left|\varepsilon_{\tau e}^{e L}\right|<0.4 \quad\left|\varepsilon_{\tau e}^{e R}\right|<0.7
$$




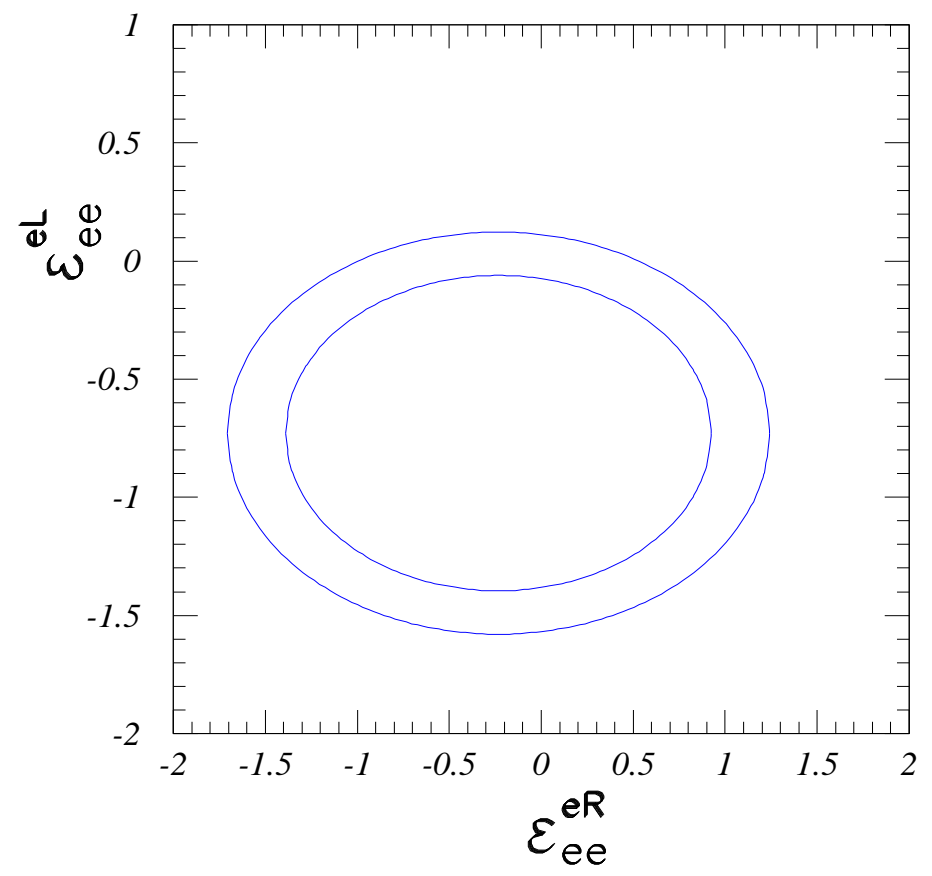

Figure 1: Bounds on flavor conserving non-standard $\nu_{e} e$ interactions from LSND experiment. Allowed regions at $90 \%$ CL are between the two ellipses.

One can wonder how these bounds would be relaxed when allowing for several operators to be present simultaneously. We consider then both, left- and right-handed diagonal NSI. The result is shown in Fig. 1. The $90 \%$ $\mathrm{CL}$ allowed region is between the two ellipses, and corresponds to the range

$$
0.445<\left(0.7282+\varepsilon_{e e}^{e L}\right)^{2}+\frac{1}{3}\left(0.2326+\varepsilon_{e e}^{e R}\right)^{2}<0.725
$$

- $\nu_{e} q \rightarrow \nu q$ scattering

The CHARM collaboration measured the following combination of $\nu_{e} N$ and $\bar{\nu}_{e} N$ cross sections [28]:

$$
R^{e}=\frac{\sigma\left(\nu_{e} N \rightarrow \nu X\right)+\sigma\left(\bar{\nu}_{e} N \rightarrow \bar{\nu} X\right)}{\sigma\left(\nu_{e} N \rightarrow e X\right)+\sigma\left(\bar{\nu}_{e} N \rightarrow \bar{e} X\right)}=\left(\tilde{g}_{L e}\right)^{2}+\left(\tilde{g}_{R e}\right)^{2}=0.406 \pm 0.140
$$

Since charged current NSI are strongly constrained, we neglect them and use this measurement to bound the neutrino neutral current NSI. In this case the effective couplings $\left(\tilde{g}_{L e}\right)^{2},\left(\tilde{g}_{R e}\right)^{2}$ are given by

$$
\begin{aligned}
& \left(\tilde{g}_{L e}\right)^{2}=\left(g_{L}^{u}+\varepsilon_{e e}^{u L}\right)^{2}+\sum_{\alpha \neq e}\left|\varepsilon_{\alpha e}^{u L}\right|^{2}+\left(g_{L}^{d}+\varepsilon_{e e}^{d L}\right)^{2}+\sum_{\alpha \neq e}\left|\varepsilon_{\alpha e}^{d L}\right|^{2} \\
& \left(\tilde{g}_{R e}\right)^{2}=\left(g_{R}^{u}+\varepsilon_{e e}^{u R}\right)^{2}+\sum_{\alpha \neq e}\left|\varepsilon_{\alpha e}^{u R}\right|^{2}+\left(g_{L}^{d}+\varepsilon_{e e}^{d R}\right)^{2}+\sum_{\alpha \neq e}\left|\varepsilon_{\alpha e}^{d R}\right|^{2} .
\end{aligned}
$$

The SM couplings corresponding to the best fit are $\left(\tilde{g}_{L e}\right)^{2}=0.3042$ and $\left(\tilde{g}_{R e}\right)^{2}=0.0301$. Using this result, the $90 \%$ CL bounds on flavour diagonal NSI are

$$
\begin{aligned}
-1 . & <\varepsilon_{e e}^{u L}<0.3 \\
-0.3<\varepsilon_{e e}^{d L} & <0.3 \\
-0.4 & <\varepsilon_{e e}^{u R}<0.7 \\
-0.6 & <\varepsilon_{e e}^{d R}<0.5
\end{aligned}
$$

when assuming only one operator at a time.

The corresponding $90 \%$ CL bounds for flavour changing NSI interactions are

$$
\left|\varepsilon_{\tau e}^{q P}\right|<0.5 \quad q=u, d \quad P=L, R
$$


Again, these bounds are only relevant for $\nu_{e} \nu_{\tau}$, since for $\nu_{e} \nu_{\mu}$ tighter ones are derived from one loop effects.

If we consider all kind of diagonal NSI, the allowed regions at 90\% CL are limited by two four dimensional ellipsoids and are given by

$$
0.176<\left(0.3493+\varepsilon_{e e}^{u L}\right)^{2}+\left(-0.4269+\varepsilon_{e e}^{d L}\right)^{2}+\left(-0.1551+\varepsilon_{e e}^{u R}\right)^{2}+\left(0.0775+\varepsilon_{e e}^{d R}\right)^{2}<0.636
$$

- $\nu_{\mu} e \rightarrow \nu e$

The CHARM II collaboration gives the following results for vector and axial-vector $e-\nu_{\mu}$ couplings [29]:

$$
g_{V}^{e}=-0.035 \pm 0.017 \quad \text { and } \quad g_{A}^{e}=-0.503 \pm 0.017
$$

where they have used LEP forward-backward asymmetry to determine the signs. From these one gets

$$
g_{L}^{e}=-0.269 \pm 0.017 \quad \text { and } \quad g_{R}^{e}=0.234 \pm 0.017
$$

The SM values of the left- and right-handed couplings are the same as for $\nu_{e} e$ scattering, so we can readily derive the $90 \%$ CL bounds on diagonal NSI

$$
\begin{aligned}
& -0.025<\varepsilon_{\mu \mu}^{e L}<0.03 \\
& -0.027<\varepsilon_{\mu \mu}^{e R}<0.03
\end{aligned}
$$

as well as on flavour changing operators,

$$
\left|\varepsilon_{\tau \mu}^{e P}\right|<0.1 \quad P=L, R
$$

when we allow only flavor changing NSI.

- $\nu_{\mu} q \rightarrow \nu q$

The NuTeV collaboration measures the ratios of neutral current to charged current neutrino-nucleon cross sections, which for an isoscalar target and at leading order are given by

$$
\begin{aligned}
& R_{\nu} \equiv \frac{\sigma(\nu N \rightarrow \nu X)}{\sigma(\nu N \rightarrow \mu X)}=\left(\tilde{g}_{L \mu}\right)^{2}+r\left(\tilde{g}_{R \mu}\right)^{2} \\
& R_{\bar{\nu}} \equiv \frac{\sigma(\bar{\nu} N \rightarrow \bar{\nu} X)}{\sigma(\bar{\nu} N \rightarrow \bar{\mu} X)}=\left(\tilde{g}_{L \mu}\right)^{2}+\frac{1}{r}\left(\tilde{g}_{R \mu}\right)^{2}
\end{aligned}
$$

where

$$
r=\frac{\sigma(\bar{\nu} N \rightarrow \bar{\mu} X)}{\sigma(\nu N \rightarrow \mu X)} .
$$

Neglecting charged current NSI, the effective couplings $\left(\tilde{g}_{L \mu}\right)^{2},\left(\tilde{g}_{R \mu}\right)^{2}$ are as given in eqs.(11),(12), just changing $e \rightarrow \mu$ in the coefficients of the neutral current NSI. The values of these couplings reported by NuTeV are [30]

$$
\left(\tilde{g}_{L \mu}\right)^{2}=0.3005 \pm 0.0014 \quad \text { and } \quad\left(\tilde{g}_{R \mu}\right)^{2}=0.0310 \pm 0.0011 .
$$

While $\left(\tilde{g}_{R \mu}\right)^{2}$ is in agreement with the SM, $\left(\tilde{g}_{R \mu}\right)_{S M}^{2}=0.0301,\left(\tilde{g}_{L \mu}\right)^{2}$ is about $3 \sigma$ away from the SM prediction, $\left(\tilde{g}_{L \mu}\right)_{S M}^{2}=0.3042$.

The $\mathrm{NuTeV}$ result for $\left(\tilde{g}_{L \mu}\right)^{2}$ can be fitted (at $90 \%$ CL) by

$$
-0.009<\varepsilon_{\mu \mu}^{u L}<-0.003 \quad \text { or } \quad 0.002<\varepsilon_{\mu \mu}^{d L}<0.008
$$

and, in principle, since the measured $\left(\tilde{g}_{L \mu}\right)^{2}$ is smaller than the SM prediction, pure left-handed flavour changing NSI are excluded because they do not interfere with the SM amplitude and therefore always give a positive contribution.

Alternatively one can assume some other explanation [31] of the discrepancy, and estimate that NSI should contribute less than $1.64 \sigma$ to the $\mathrm{NuTeV}$ result. This leads to the constraints:

$$
\begin{array}{rl}
\left|\varepsilon_{\mu \mu}^{q L}\right|<0.003 & q=u, d \\
\left|\varepsilon_{\tau \mu}^{q L}\right|<0.05 & q=u, d
\end{array}
$$

For diagonal right-handed NSI the $90 \%$ CL allowed ranges are

$$
\begin{gathered}
-0.008<\varepsilon_{\mu \mu}^{u R}<0.003 \\
-0.008<\varepsilon_{\mu \mu}^{d R}<0.015
\end{gathered}
$$



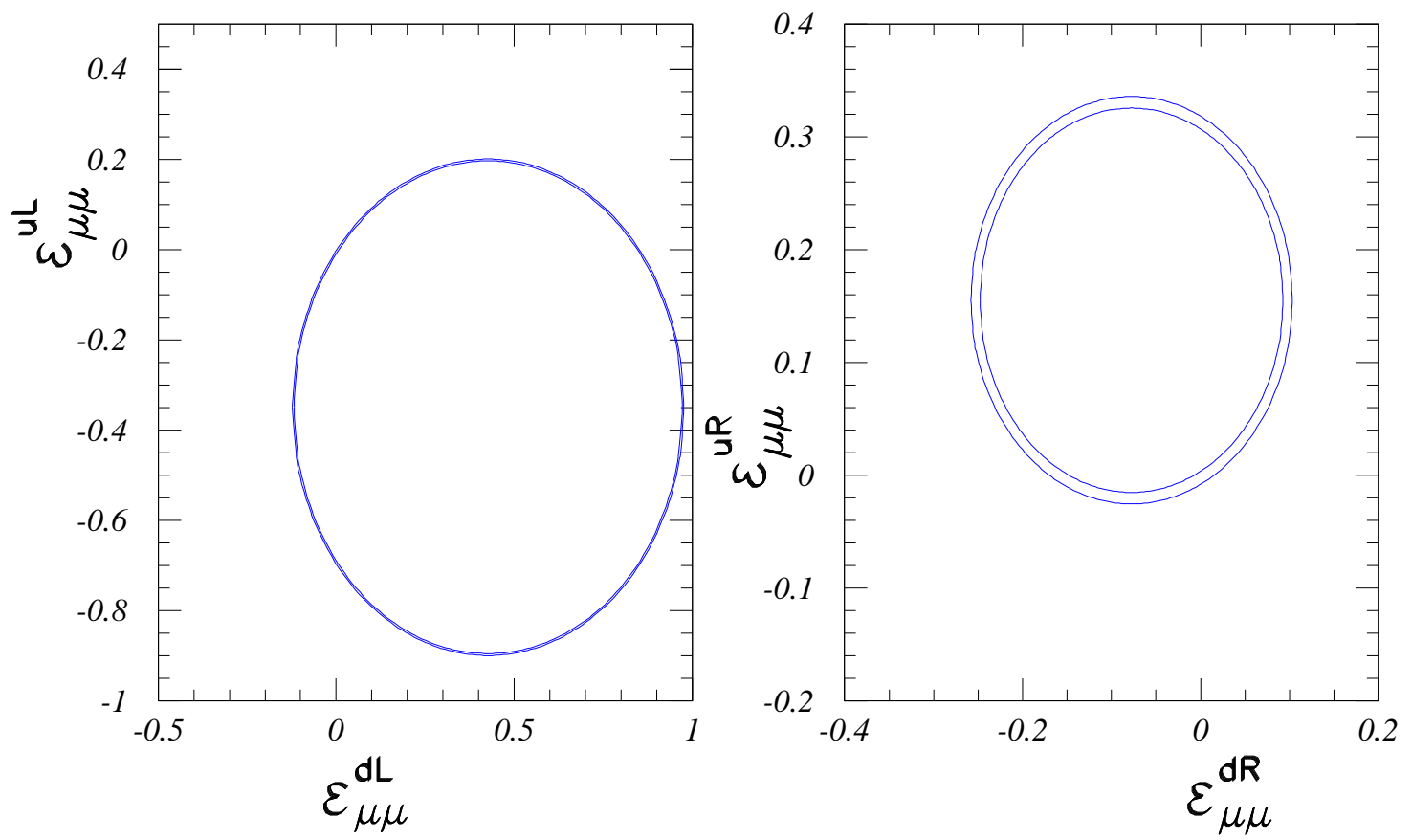

Figure 2: Bounds on flavor conserving non-standard $\nu_{\mu} q$ interactions from NuTeV experiment. The $90 \%$ CL allowed regions are the thick ellipse in the left panel and the region between the two ellipses in the right one.

while for flavour changing interactions,

$$
\left|\varepsilon_{\tau \mu}^{q R}\right|<0.05 . \quad q=u, d
$$

These bounds are relaxed if we consider several operators present simultaneously. In Fig. 2 we plot the $90 \%$ CL limits on diagonal neutrino NSI with $\mathrm{u}$ - and d-type quarks, both for left- and right-handed interactions. The allowed regions are the thick ellipse in the left panel $\S$ and the region between the two ellipses in the right one. They are described by the equations:

$$
\begin{array}{ll}
0.2982<\left(0.3493+\varepsilon_{\mu \mu}^{u L}\right)^{2}+\left(-0.4269+\varepsilon_{\mu \mu}^{d L}\right)^{2} & <0.3028 \\
0.0292<\left(-0.1551+\varepsilon_{\mu \mu}^{u R}\right)^{2}+\left(0.0775+\varepsilon_{\mu \mu}^{d R}\right)^{2} & <0.0328
\end{array}
$$

\subsubsection{LEP}

The authors of Ref. [18] have pointed out the importance of the $e^{+} e^{-} \rightarrow \nu \bar{\nu} \gamma$ cross section measured at LEP II in order to constraint neutrino NSI. For the case of diagonal $\nu_{\tau} e$ interactions these are the only laboratory bounds, and for $\nu_{e} e$ they are comparable to the LSND limits already discussed. We refer the reader to [18] for the detailed analysis, and just summarize here the $90 \%$ CL bounds when only $\nu_{\tau} e$ NSI are considered, which can be read from their Fig. 4: ब

$$
\begin{aligned}
& -0.6<\varepsilon_{\tau \tau}^{e L}<0.4 \\
& -0.4<\varepsilon_{\tau \tau}^{e R}<0.6
\end{aligned}
$$

This reaction is also useful to constrain flavor changing NSI, but the bounds are comparable or looser than the ones derived from elastic scattering, namely

$$
\left|\varepsilon_{\alpha \beta}^{e P}\right|<0.4 \quad P=L, R, \alpha=\tau, \beta=e, \mu
$$

$\S$ There are indeed two ellipses, but they can not be distinguished in the figure.

๑ We have estimated $\mathrm{a} \sim 10 \%$ accuracy at $90 \%$ CL. 
assuming only flavor changing non-standard operators.

This process cannot constrain $\nu_{\tau} q$ interactions. However, it is obvious that any NSI interaction can contribute to $Z \rightarrow f \bar{f} \nu \bar{\nu}$ at LEP1 or $e e^{+} \rightarrow f \bar{f} \nu \bar{\nu}$ at LEP2. These processes occur in the SM with a pair of virtual $Z, W, \gamma$. They have also been observed and the observations roughly agree with the SM expectations. For instance the cross section with virtual $Z$ and $\gamma^{*}$ going to quarks and neutrinos $e^{+} e^{-} \rightarrow Z \gamma^{*} \rightarrow q q \nu \nu$ has been measured by DELPHI at energies above $189 \mathrm{GeV}$ to be $\sigma_{Z \gamma^{*}}=(0.129 \pm 0.038)$ which has to be compared with the SM prediction of about 0.092-0.084. This suggest that NSI should be at most as strong as SM interactions. It is difficult to extract more precise information on possible NSI from these data because it is based on a particular pole structure which is not shared by the NSI. Clearly, to be more precise on the NSI a dedicated study should be performed. In addition, NSI of $\nu_{\tau}$ with quarks can in principle be constrained through one loop effects both in the invisible and the hadronic Z width, but typically these bounds are $\mathcal{O}(1)$ (see sect. 3.2).

One can ask whether TESLA would set improved bounds. The sensitivity at TESLA to contact interactions of the form $\eta 2 \sqrt{2} G_{F}\left(\bar{e} \gamma^{\rho} P e\right)\left(\bar{\tau} \gamma_{\rho} \mu, e\right)$ can be estimated [32] to be $\eta \gtrsim 0.5 \times 10^{-3}$. Using $c \sim .002$ from section 3.2, $\eta \sim c \varepsilon$ implies $\varepsilon<O(0.3)$. This is not particularly significant, and corresponds to a new physics mass scale below $500 \mathrm{GeV}(\sqrt{s}$ at TESLA) so the contact interaction analysis is not appropriate. A more promising channel might be $e \bar{e} \rightarrow \gamma(\nu \bar{\nu})$, but to our knowledge, the limits that could be extracted from searching for this at TESLA have not been studied.

\subsubsection{Atmospheric neutrinos}

Exotic (i.e., no-oscillation) solutions of the atmospheric neutrino problem are disfavored by the energy and baseline dependence [3,4]. In particular, in Ref.[5] an explanation of atmospheric neutrino data in terms of pure NSI of neutrinos is ruled out at $99 \% \mathrm{CL}$, but a combined analysis is performed which includes both, oscillations and nonstandard neutrino-matter interactions, and allows to set significant bounds on diagonal as well as flavour changing neutrino's NSI. Two comments are in order. First, note that such bounds apply to the vector coupling constant of the NSI, $\varepsilon_{\alpha \beta}^{f V}=\varepsilon_{\alpha \beta}^{f L}+\varepsilon_{\alpha \beta}^{f R}$, since it is the only one which appears in neutrino propagation through matter. Second, the study does not consider NSI in the production neither in the detection of the neutrinos. However both processes take place through charged current interactions, which are better constrained, so one does not expect sizable effects.

Notice that when NSI appear only in the propagation of neutrinos in matter, the relevant parameters are the flavour changing NSI couplings and the difference between the diagonal ones, denoted in $[5,33]$ as the non-universality parameter $\varepsilon^{\prime}$. The combined analysis leads to the following 90\% CL bounds when both diagonal and flavour changing NSI are simultaneously present [33]:

$$
\begin{gathered}
-0.016<\varepsilon_{\mu \tau}^{d V}<-0.009 \quad 0<\varepsilon_{\mu \tau}^{d V}<0.013 \\
\left|\varepsilon_{\mu \mu}^{d V}-\varepsilon_{\tau \tau}^{d V}\right|<0.03
\end{gathered}
$$

Although the fit has been done assuming only neutrino-down quark NSI similar results can be expected for the neutrino-electron and neutrino-up quark cases.

This result leads to a stringent constraint on flavour changing four-fermion operators involving $\nu_{\mu} \nu_{\tau}$, and provides a complementary bound on diagonal NSI of $\nu_{\tau}$ with quarks, only loosely constrained by LEP.

\section{$3.2 \quad$ One loop effects}

On general grounds we expect that interactions in which the $\nu_{\alpha}$ are replaced by the corresponding leptons will be generated by one loop diagrams with virtual $W$ 's. Effective interactions, however, are nonrenormalizable and, therefore, a precise prescription has to be given in order to estimate these corrections. Our point of view will be that these are originated from a more complete theory at scales $\Lambda \gg m_{W}$ which is renormalizable (or perhaps finite) and in which observables can be computed in terms of a few parameters.

We illustrate our point with a simple toy model, in which exact loop calculations can be easily done. It is not a realistic example of the type of model we wish to constrain (see e.g. [18] for such models), because it induces $\left(\bar{e} \gamma^{\rho} P e\right)\left(\bar{\nu}_{\mu} \gamma_{\rho} L \nu_{\tau}\right)$ and $\left(\bar{\tau} \gamma^{\rho} P e\right)\left(\bar{\nu}_{e} \gamma_{\rho} L \nu_{\mu}\right)$ simultaneously at dimension 6 with approximately the same coefficient. So $\tau$ appearance at the near detector of a neutrino factory would be more sensitive to this model than neutrino scattering.

As a guide for the type of calculations we are going to perform, we imagine the standard model extended by a singly charged scalar singlet $h^{+}$, with mass $M$, which has the following interaction with the standard leptonic doublet, 


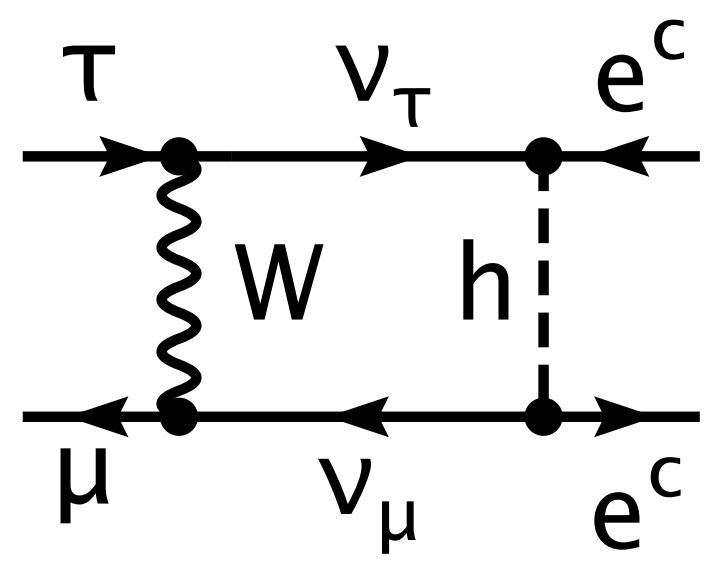

Figure 3: One-loop contributions to four-fermion interactions in a theory with a charged scalar singlet.

$\ell,\left(\tilde{\ell}=i \tau_{2} \ell^{c}\right)$

$$
\mathcal{L}_{h}=f_{\alpha \beta}\left(\overline{\ell_{\alpha}} \tilde{\ell}_{\beta}\right) h^{-}+\text {h.c. },
$$

where $f_{\alpha \beta}$ is a coupling antisymmetric in flavour indices. Expanding in the fields

$$
\mathcal{L}_{h}=2 f_{\alpha \beta}\left(\overline{\nu_{L \alpha}} e_{L \beta}^{c}\right) h^{-}+\text {h.c. . }
$$

Exchange of scalars will generate a four-fermion interaction of the type we are considering (for a review of this model from the effective Lagrangian point of view see [34]),

$$
\mathcal{L}=4 f_{\alpha \beta} f_{\gamma \delta}^{*} \frac{1}{M^{2}}\left(\overline{e_{L \delta}^{c}} \nu_{L \gamma}\right)\left(\bar{\nu}_{L \alpha} e_{L \beta}^{c}\right)=2 f_{\alpha \beta} f_{\gamma \delta}^{*} \frac{1}{M^{2}}\left(\overline{e_{\beta}} \gamma^{\rho} L e_{\delta}\right)\left(\bar{\nu}_{\alpha} \gamma_{\rho} L \nu_{\gamma}\right)
$$

We are only interested in NSI with electrons, so we take $f_{\mu \tau}=0$ and find that this model gives

$$
\mathcal{L}=-2 \sqrt{2} G_{F} \varepsilon_{\mu \tau}^{e L}\left(\bar{e} \gamma^{\rho} L e\right)\left(\bar{\nu}_{\mu} \gamma_{\rho} L \nu_{\tau}\right)+\text { h.c. }+\cdots,
$$

where

$$
\varepsilon_{\mu \tau}^{e L}=-\frac{f_{\mu e} f_{\tau e}^{*}}{g^{2}} 4 \frac{m_{W}^{2}}{M^{2}} .
$$

In addition this model also generates, at tree level, interactions of the type $\left(\bar{e} \gamma^{\rho} L \tau\right)\left(\bar{\nu}_{\mu} \gamma_{\rho} L \nu_{e}\right)$ and $\left(\bar{\mu} \gamma^{\rho} L \tau\right)\left(\bar{\nu}_{e} \gamma_{\rho} L \nu_{e}\right)$ which are not interesting for our discussion and which we have represented by the dots in eq. (43).

Although at tree level this model does not provide any contribution to $\tau^{-} \rightarrow \mu^{-} e^{-} e^{+}$it does contain contributions at the one-loop level. The only contribution with $\nu_{\mu}$ and $\nu_{\tau}$ in virtual states is given by the diagram in Fig. 3 (note that in this model there are other diagrams contributing to the process with other types of neutrinos in virtual states). In fact the diagram in Fig. 3 can be easily computed and it is finite. In the limit of $M_{W}, M \gg m_{\tau}$ it generates the following interaction among four charged leptons (after Fierz reordering)

$$
\mathcal{L}=-2 \sqrt{2} G_{F} \varepsilon_{\mu \tau}^{e L} \frac{\alpha}{8 \pi s_{W}^{2}} F\left(\frac{M^{2}}{m_{W}^{2}}\right)\left(\bar{e} \gamma^{\rho} L e\right)\left(\bar{\mu} \gamma_{\rho} L \tau\right)
$$

where $\varepsilon_{\mu \tau}^{e L}$ is given by eq. (44) and the function $F(x)$ is

$$
F(x)=\frac{x}{x-1} \ln (x) \approx \ln (x) .
$$

The important thing is that to obtain this logarithmic contribution we do not need to know all the details of the complete theory. We can compute it by using the four-fermion effective interaction (Fig. 4).

This diagram yields a logarithmically divergent contribution. However, the divergent part can be unambiguously calculated. In the effective field theory language one should add a counter-term (a 4-charged fermion interaction) to the Lagrangian in order to absorb this divergence. The coefficient of this new operator will run under the influence of the diagram in Fig. 4. Therefore even if at the scale of new physics, $\Lambda$, there is no 4 -charged fermion interaction at tree level it will be generated through running from the scale $\Lambda$ to the electroweak scale. Thus, to compute the logarithmic contribution it is enough to compute the divergent part of the diagram in Fig. 4. The finite parts have to do with possible non-logarithmic contributions generated at the scale $\Lambda$ which cannot be computed without knowing 


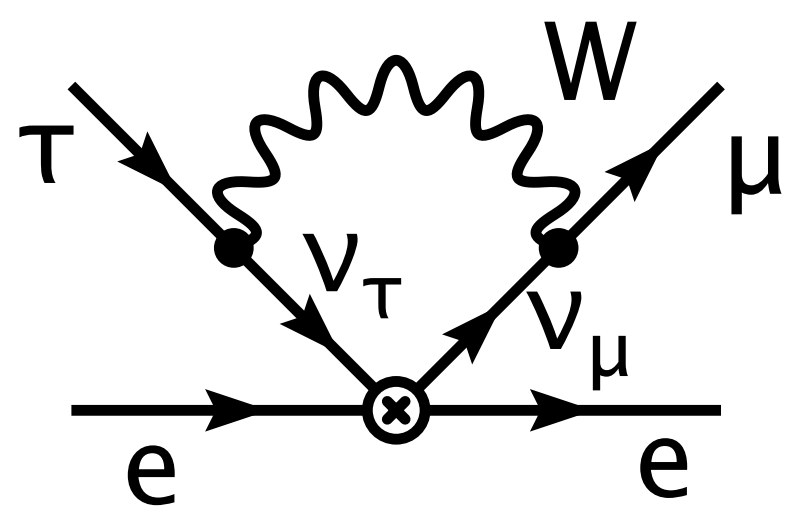

Figure 4: One-loop contributions to four-fermion interactions in the effective theory.

the details of the complete theory and should be absorbed in the initial conditions for the running of the different operators. However, if the scale $\Lambda$ is much larger than the electroweak scale, one can reasonably assume that the logarithm $\ln \left(\Lambda / m_{W}\right)$ dominates completely the result.

If we apply this point of view to the model we just considered we obtain exactly the same result as in eq. (45) with $F\left(M^{2} / m_{W}^{2}\right)$ replaced by $2 \ln \left(\Lambda / m_{W}\right)+\kappa(\Lambda)$, where $\kappa(\Lambda)$ takes into account possible non-logarithmic contributions at the scale $\Lambda$. So the effective Lagrangian calculation gives the correct answer if we identify $\Lambda$ with the mass of the charged singlet, $M$ and take $\kappa(M)=0$. Note, however, that in the effective field theory calculation we have no way to determine $\kappa(\Lambda)$, which depends on the details of the complete theory. In fact, in the effective field theory $\Lambda$ can only loosely be related to the masses of the unknown more complete theory. $\kappa(\Lambda)$, somehow, parameterizes all these unknown details. The important point is that we expect it to be at most order 1 and negligible in front of the calculable logarithmic piece if $\Lambda$ is large enough.

In order to set reliable bounds on the $\varepsilon$ 's using these one loop corrections we would need to know roughly the size of $\Lambda$. However this is not known. Bounds will be set directly on the $\varepsilon$ 's at tree level and on $\varepsilon \ln \left(\Lambda / m_{W}\right)$ at one loop. Of course, $\varepsilon$ also depends implicitly on $m_{W}^{2} / \Lambda^{2}$ as in eq. (44), however, it also depends on other parameters, in this case the Yukawa and gauge couplings, which, at the level of the effective four-fermion theory cannot be completely disentangled. One might expect the logarithm to give some enhancement, but, since the size of this enhancement cannot be reliably computed we choose to be conservative and take $\ln \left(\Lambda / m_{W}\right) \approx 1$ in all the bounds we will set from loop calculations.

\subsubsection{Limits on lepton flavor violating interactions}

Using these arguments we can get some indirect bounds on the interactions in eq. (2) by using radiative corrections. The calculation of the diagram in Fig. 4 remains essentially unchanged if we use as starting point an effective interaction with other type of neutrinos or with neutrinos and quarks, thus, if we have an interaction among electrons, u-quarks or d-quarks with neutrinos with strengths $\varepsilon_{\alpha \beta}^{e P}, \varepsilon_{\alpha \beta}^{u P}, \varepsilon_{\alpha \beta}^{d P}$ respectively, we expect a four-fermion interaction among electrons, u-quarks or d-quarks with the charged leptons with strengths $c \varepsilon_{\alpha \beta}^{e P}$, c $\varepsilon_{\alpha \beta}^{u P}$, c $\varepsilon_{\alpha \beta}^{d P}$ with

$$
c=\frac{\alpha}{4 \pi s_{W}^{2}} \ln \left(\frac{\Lambda}{m_{W}}\right) \approx 0.0027 .
$$

These interactions give rise to a class of interesting processes like $\mu^{-} \rightarrow e^{+} e^{-} e^{-}\left(B R<1.0 \times 10^{-12}\right), \tau^{-} \rightarrow e^{+} e^{-} e^{-}$ $\left(B R<2.9 \times 10^{-6}\right), \tau^{-} \rightarrow e^{+} e^{-} \mu^{-}\left(B R<1.7 \times 10^{-6}\right), \mu T i \rightarrow e T i\left(\Gamma<4.3 \times 10^{-12}\right), \tau^{-} \rightarrow e^{-} \pi^{0}\left(B R<3.7 \times 10^{-6}\right)$, $\tau^{-} \rightarrow \mu^{-} \pi^{0}\left(B R<4.0 \times 10^{-6}\right), \tau^{-} \rightarrow e^{-} \rho^{0}\left(B R<2.0 \times 10^{-6}\right), \tau^{-} \rightarrow \mu^{-} \rho^{0}\left(B R<6.3 \times 10^{-6}\right)$ which are strongly bounded from present experiments, thus one can obtain some information on $\varepsilon_{e \mu}^{e P}, \varepsilon_{e \tau}^{e P}, \varepsilon_{\mu \tau}^{e P}$, and similarly for quark interactions with neutrinos. We have listed the branching ratios we use to set bounds, so that the limits on the $\varepsilon$ 's can be rescaled if the experimental bounds become stronger.

Consider for example the bound on $\varepsilon_{\tau \mu}^{e P}$ from $\tau^{-} \rightarrow \mu^{-} e^{+} e^{-}$. The diagram of Fig. 4 with the vertex $\otimes$ given by eq. (2) provides an interaction (like that in eq. (45))

$$
\mathcal{L}=-2 \sqrt{2} G_{F} c \varepsilon_{\mu \tau}^{e P}\left(\bar{e} \gamma^{\rho} P e\right)\left(\bar{\mu} \gamma_{\rho} L \tau\right)
$$


with $c$ given in eq. (46). From this we can immediately compute the branching ratio

$$
B R\left(\tau^{-} \rightarrow \mu^{-} e^{+} e^{-}\right)=B R\left(\tau^{-} \rightarrow \mu^{-} \nu_{\tau} \bar{\nu}_{\mu}\right) \frac{\Gamma\left(\tau^{-} \rightarrow \mu^{-} e^{+} e^{-}\right)}{\Gamma\left(\tau^{-} \rightarrow \mu^{-} \nu_{\tau} \bar{\nu}_{\mu}\right)}=0.1737\left|c \varepsilon_{\tau \mu}^{e P}\right|^{2}<1.7 \times 10^{-6},
$$

from where we obtain

$$
\left|\varepsilon_{\tau \mu}^{e P}\right|<0.0031 / c<1.2, \quad 90 \% \text { CL }
$$

Note that, as can easily be checked from the Michel parameters, the operators $\left(\bar{\ell}_{\beta} \gamma^{\rho} \ell_{\alpha}\right)\left(\bar{e} \gamma_{\rho} L e\right)$ and $\left(\bar{\ell}_{\beta} \gamma^{\rho} \ell_{\alpha}\right)$ $\left(\bar{e} \gamma_{\rho} R e\right)$ give the same contribution to the total decay rate.

Somehow worse bounds can be obtained for $\varepsilon_{\tau e}^{e P}$, $\left|\varepsilon_{\tau e}^{e P}\right|<2.9$, because the limits on $\tau^{-} \rightarrow e^{-} e^{+} e^{-}$are a bit looser and because of the two identical particles in the final state.

Much more interesting, however, are the bounds that can be set on $\varepsilon_{\mu e}^{e P}$ because of the strong experimental limit on $B R\left(\mu^{-} \rightarrow e^{-} e^{+} e^{-}\right)$. In this case the calculation is similar except for a few factors due to the identical particles in the final state. We have

$$
B R\left(\mu^{-} \rightarrow e^{-} e^{+} e^{-}\right) \approx \frac{\Gamma\left(\mu^{-} \rightarrow e^{-} e^{+} e^{-}\right)}{\Gamma\left(\mu^{-} \rightarrow e^{-} \nu_{\mu} \bar{\nu}_{e}\right)}=0.5\left|c \varepsilon_{\mu e}^{e P}\right|^{2}<1 \times 10^{-12},
$$

from where we obtain

$$
\left|\varepsilon_{\mu e}^{e P}\right|<1.4 \times 10^{-6} / c<5 \times 10^{-4}, \quad 90 \% \mathrm{CL} .
$$

Similar arguments can be used for NSI of quarks with neutrinos. In fact, Fig. 4 also generates interactions like eq. (45) but with the electron fields replaced by $u$ or $d$-quark fields. These interactions contribute to several hadronic decays of the tau lepton. Thus, we can set bounds on the $\varepsilon_{\tau \beta}^{q P}$ (with $\beta=e, \mu$ and $q=u, d$ ). For instance, assuming no unnatural cancellations among the $u$-quark and the $d$-quark or the $P=L$ and $P=R$ contributions, we have

$$
\left|\varepsilon_{\tau e}^{q P}\right|<\sqrt{2} / c \sqrt{\frac{B R\left(\tau^{-} \rightarrow e^{-} \rho^{0}\right)}{B R\left(\tau^{-} \rightarrow \nu_{\tau} \rho^{-}\right)}}<1.6,
$$

where we included the isospin factor $\sqrt{2}$ and used $B R\left(\tau^{-} \rightarrow \nu_{\tau} \rho^{-}\right) \approx 0.22$.

From the decay $\tau^{-} \rightarrow e^{-} \pi^{0}$ one obtains similar but slightly worse bounds because the limit on $B R\left(\tau^{-} \rightarrow e^{-} \pi^{0}\right)$ is worse and because $B R\left(\tau^{-} \rightarrow \nu_{\tau} \pi^{-}\right)$is smaller. It is important, however, to remark that decays into $\rho$ 's probe the vector channel and decays into $\pi$ 's probe the pseudoscalar channel and, in this sense, provide a complementary information.

Using $\tau^{-} \rightarrow \mu^{-} \rho^{0}$ (or $\tau^{-} \rightarrow \mu^{-} \pi^{0}$ ) one can also set bounds on $\varepsilon_{\tau \mu}^{q P}$. Using decays into $\rho$ 's we obtain $\left|\varepsilon_{\tau \mu}^{q P}\right|<2.8$.

Finally we can set bounds on $\varepsilon_{\mu e}^{q P}$ from $\mu-e$ conversion on nuclei. Comparing the experimental upper bound on the rate of $\mu-e$ conversion to the rate of muon capture,

$$
R_{\mu e} \equiv \frac{\sigma\left(\mu^{-} T i \rightarrow e^{-} T i\right)}{\sigma\left(\mu^{-} T i \rightarrow \text { capture }\right)}<4.3 \times 10^{-12},
$$

implies (see e.g. [35]) that

$$
\varepsilon_{\mu e}^{q P} \lesssim \sqrt{R_{\mu e}} / c \approx 7.7 \times 10^{-4} .
$$

We have collected the numerical values of the best bounds in table 3 .

\subsubsection{Limits on lepton flavor conserving interactions}

For lepton flavor conserving operators, diagrams like Fig. 4 also give rise to interactions with four charged leptons, however the information we can extract from them is not so useful. In addition, in that case, there are other interesting interactions that can also be generated through radiative corrections. In particular, loop corrections involving interactions like the ones in eq. (2) can affect in a non-universal way the decay rates of the electroweak gauge bosons as shown in Figs. 5-7. This type of interactions also occur in the SM but in the SM they are universal, they have the same strength for the different generations. Then, we can bound them by using the various universality tests. These are checked, at most, at the level of $0.1 \%$, which is of the order of the SM radiative corrections (apart from $m_{t}$ corrections or running $\alpha$ corrections) which are order $\frac{\alpha}{\pi}$. We expect the one-loop contributions from NSI to give corrections $\frac{\alpha}{\pi} \varepsilon$ therefore we expect, from these processes, to obtain very weak bounds $\varepsilon<1$. Let us see how these bounds arise. 


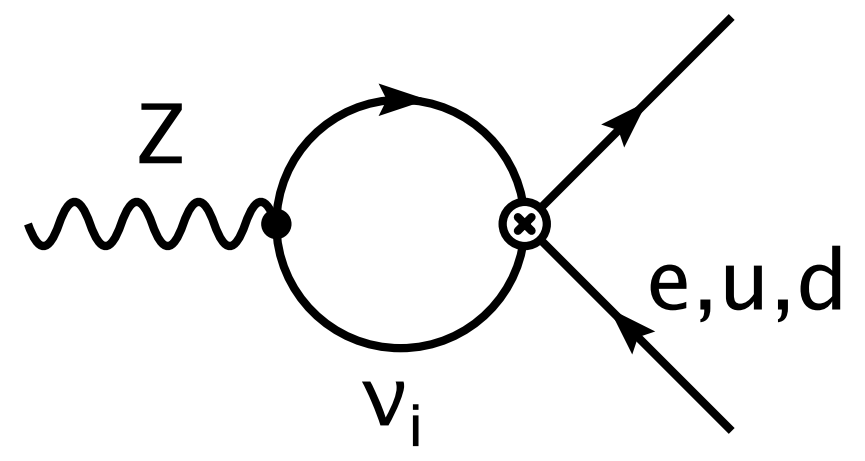

Figure 5: Contributions of lepton flavor conserving NSI to the vertex of the Z-gauge bosons to electrons, $u$ and $d$-quarks.

Fig. 5 gives corrections to the hadronic and leptonic decay widths of the $Z$-boson. The NSI contributions to the amplitudes of these processes interfere with the tree-level SM amplitudes and the total correction is proportional to the sum of all neutrino couplings. Since $\varepsilon_{e e}^{f P}, \varepsilon_{\mu \mu}^{f P}$ are bounded by other means we will consider only $\nu_{\tau}$ interactions. The calculation of the diagram gives a correction to the couplings of of $e, u, d$ given by $(f=e, u, d)$

$$
\begin{gathered}
\delta g_{V}^{f}=\frac{\alpha}{12 \pi s_{W}^{2} c_{W}^{2}} \ln \left(\frac{\Lambda}{m_{Z}}\right) \sum_{\alpha=e, \mu, \tau} \sum_{P} \varepsilon_{\alpha \alpha}^{f P}, \\
\delta g_{A}^{f}=\frac{\alpha}{12 \pi s_{W}^{2} c_{W}^{2}} \ln \left(\frac{\Lambda}{m_{Z}}\right) \sum_{\alpha=e, \mu, \tau} \sum_{P}(-1)^{P} \varepsilon_{\alpha \alpha}^{f P},
\end{gathered}
$$

where we have adopted the PDG convention for SM $Z$-couplings to fermions,

$$
\mathcal{L}_{Z}=-\frac{e}{2 s_{W} c_{W}} \sum_{f} \overline{\psi_{f}} \gamma^{\rho}\left(g_{V}^{f}-g_{A}^{f} \gamma_{5}\right) \psi_{f} Z_{\rho}
$$

with $g_{V}=g_{L}+g_{R}, g_{A}=g_{L}-g_{R}$ (see table 1 ), and $(-1)^{P}=+1$ for $P=L$ and $(-1)^{P}=-1$ for $P=R$. Results for $g_{V, A}^{e}$ are usually presented in terms of an effective $g_{V, A}^{e}$ at the $Z$ peak which incorporates the SM electroweak radiative corrections (but not final state QED corrections). $g_{A}^{e}$ is known with a good precision $\left(g_{A}^{e}=-0.50111 \pm 0.00035\right.$ without assuming universality in the fit) and agrees quite well with the SM prediction for a light Higgs $g_{A}^{e} \approx-0.5012$, therefore additional contributions must be small. Requiring that $\delta g_{A}^{e}$ is smaller, in absolute value, than the error, and taking $\ln \left(\Lambda / m_{Z}\right)>1$, we find

$$
\sum_{P} \sum_{\alpha=e, \mu, \tau} \varepsilon_{\alpha \alpha}^{e P}<\frac{12 \pi s_{W}^{2} c_{W}^{2}}{\alpha} 0.00035 \times 1.64 \approx 0.5,90 \% \mathrm{CL}
$$

For the vector part the precision is not so good and the agreement with the standard model is not so perfect: the measurement yields $g_{V}^{e} \approx-0.03816 \pm 0.00047$ while the $\mathrm{SM}$ prediction for a light Higgs is about $g_{V}^{e} \approx-0.037$. Requiring that the additional corrections are smaller, in absolute value, than the error we obtain

$$
\sum_{P} \sum_{\alpha=e, \mu, \tau}(-1)^{P} \varepsilon_{\alpha \alpha}^{e P}<\frac{12 \pi s_{W}^{2} c_{W}^{2}}{\alpha} 0.00047 \times 1.64 \approx 0.7,90 \% \mathrm{CL} .
$$

These bounds are comparable to the tree-level limits one can set from $e^{+} e^{-} \rightarrow \gamma \nu \bar{\nu}$ and, in particular, if we consider only couplings to $\nu_{\tau}$ and assume no cancellations among different operators we find

$$
\left|\varepsilon_{\tau \tau}^{e P}\right|<0.5
$$

If we allow for cancellations among left- and right-handed operators the limit is slightly moved to about 0.6 .

Although the same corrections appear also for light quarks, they only affect the total hadronic decay width of the $Z$, which also contains contributions from $s, c$ and $b$ quarks. One can try to subtract the $b$ and the $c$ contributions but the $s$ quark is practically impossible to separate. Therefore, although with additional assumptions (for instance that the $b, c$ and $s$ quark couplings are standard) one can set some bounds on the hadronic $\varepsilon$ 's, the bounds will not be comparable to the ones obtained for the electron couplings. 


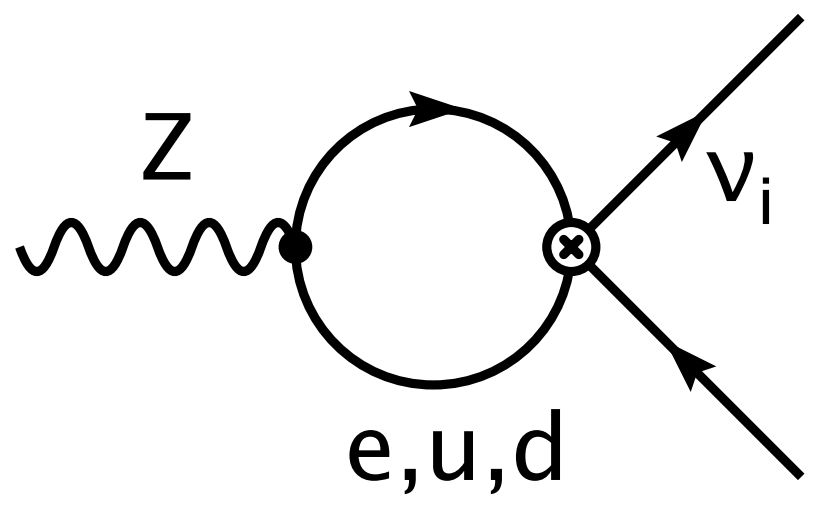

Figure 6: Contributions of lepton flavor conserving NSI to the Z invisible decay width.

On the other hand, Fig. 6 gives corrections to the invisible decay width of the $Z$-boson which, assuming 3 neutrino flavours, can be used to set bounds on the $\varepsilon$ 's. In this case non-diagonal neutrino NSI could also contribute. However, these contributions do not interfere with tree level amplitudes and are already bounded by lepton flavor violating processes. Neglecting the non-diagonal terms, Fig. 6 will be proportional to the sum of all the $\varepsilon$ 's. Evaluation of the diagram yields the following correction to the $Z$-couplings to neutrinos

$$
\delta g_{V}^{\nu_{\alpha}}=\delta g_{A}^{\nu_{\alpha}}=\frac{\alpha}{12 \pi s_{W}^{2} c_{W}^{2}} \ln \left(\frac{\Lambda}{m_{Z}}\right) \sum_{f=e, u, d} N_{C}(f) \sum_{P}\left(g_{V}^{f}+(-1)^{P} g_{A}^{f}\right) \varepsilon_{\alpha \alpha}^{f P} .
$$

Assuming that all the other couplings are standard one can obtain information on the above combination of $\varepsilon$ 's from the invisible decay width of the $Z, \Gamma_{\mathrm{i} n v}$. SM limits on $\Delta N_{\nu}$, the number of neutrino species, are in fact bounds on $\Delta \Gamma_{\mathrm{i} n v}$. If only $\nu_{\tau}$ NSI contribute to $\Gamma_{\mathrm{i} n v}\left(\nu_{e}\right.$ and $\nu_{\mu}$ interactions are already well bounded from other processes) we can set bounds on $\varepsilon_{\tau \tau}^{f P}$. Using that the $\Gamma_{\mathrm{i} n v}$ is proportional to $\sum_{\alpha}\left(g_{A}^{\nu_{\alpha}}\right)^{2}$ (we assume only left-handed neutrinos so $g_{A}^{\nu_{\alpha}}=g_{V}^{\nu_{\alpha}}$ ) one can easily see that

$$
\frac{\delta g_{A}^{\nu_{\tau}}}{g_{A}^{\nu_{\tau}}}=\frac{1}{2} \Delta N_{\nu}
$$

where in the denominator we have assumed approximate universality in the couplings. Taking only one operator at a time and requiring that the NSI contribution is smaller than the error in absolute value $\left(\Delta N_{\nu}<0.008\right)$ we find (at $90 \% \mathrm{CL})$

$$
\begin{aligned}
& \left|\varepsilon_{\tau \tau}^{u L}\right|<1.4, \\
& \left|\varepsilon_{\tau \tau}^{u R}\right|<3, \\
& \left|\varepsilon_{\tau \tau}^{d L}\right|<1.1, \\
& \left|\varepsilon_{\tau \tau}^{d R}\right|<6, \\
& \left|\varepsilon_{\tau \tau}^{e L}\right|<5, \\
& \left|\varepsilon_{\tau \tau}^{e R}\right|<6 .
\end{aligned}
$$

Of course for electron couplings to neutrinos we have much better bounds from the leptonic decay widths of the $Z$. For hadronic couplings these are the best limits we have.

In addition to the processes considered there are other one-loop diagrams that could give interesting contributions. For instance, the diagram in Fig. 7 gives corrections to the coupling of the W-boson to the electron only. This coupling can be absorbed in the definition of $G_{F}$ affecting equally $\mu$ decay and $\beta$ decays (or $\tau \rightarrow e \nu_{\tau} \bar{\nu}_{e}$ and $\pi \rightarrow e \bar{\nu}_{e}$ ). Note, however, that it will not affect $\tau \rightarrow \mu \nu_{\tau} \bar{\nu}_{\mu}$ or $\pi \rightarrow \mu \bar{\nu}_{\mu}$. Obviously, these contributions also will affect differently $W \rightarrow e \bar{\nu}_{e}$ and $W \rightarrow \mu \bar{\nu}_{\mu}$ or $W \rightarrow \tau \bar{\nu}_{\tau}$. Therefore, universality limits can be used to set bounds on $\varepsilon_{e e}^{e L}$, the only coupling appearing Fig. 7. However, we do not expect interesting bounds since this coupling is already bounded from $\nu_{e} e \rightarrow \nu_{e} e$ scattering.

Finally one can use the recent determinations of $G_{F}$ in $\tau$ decays (such as $\tau \rightarrow \nu_{\tau} \mu \bar{\nu}_{\mu}, \tau \rightarrow \nu \pi$ ) to set constraints [36] on new physics involving the $\tau$. Operators of the form $\left(\bar{\tau} \gamma^{\rho} \nu_{\tau}\right)\left(f^{\prime} \gamma_{\rho} P f\right)$ are induced at one loop by the operators we are considering (see Fig. 8). Thus, from the bounds in [36] one can extract limits on the flavour diagonal $\varepsilon_{\tau \tau}^{f L}$. However, these limits are loser than the ones already discussed and only affect left-handed couplings. 


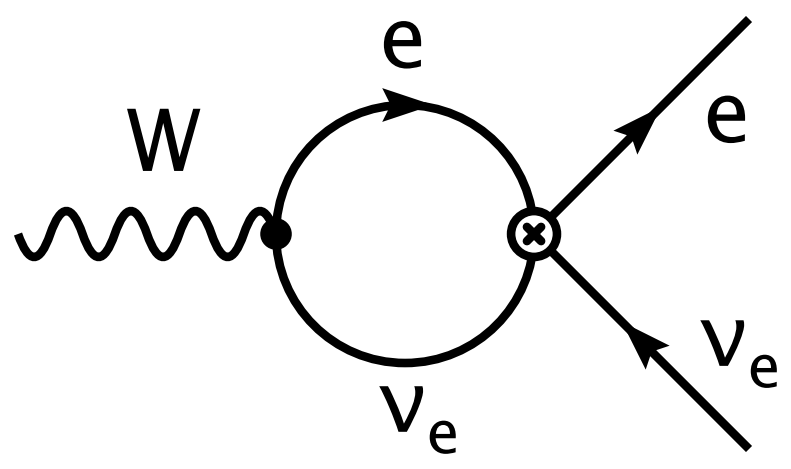

Figure 7: Contributions of leptonic flavor conserving NSI to the vertex of the W gauge boson.

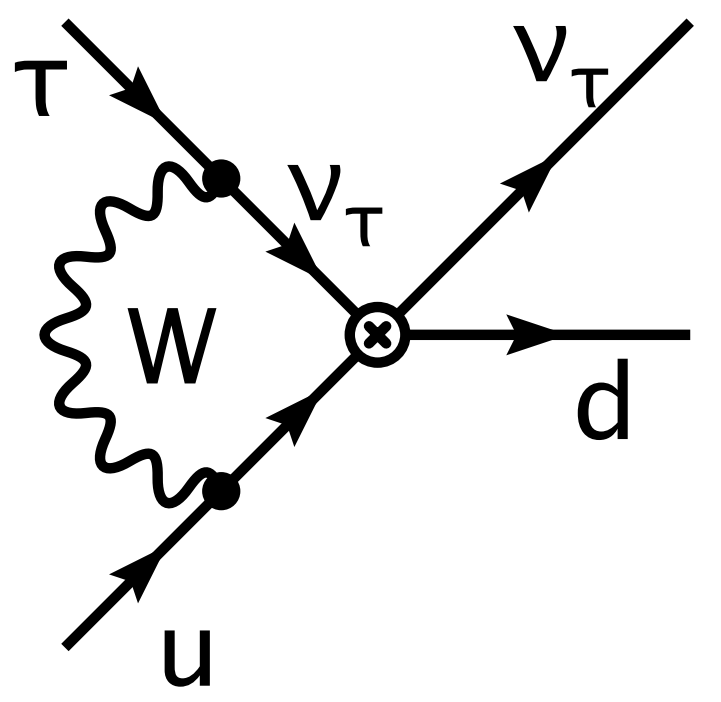

Figure 8: Contributions of lepton flavor conserving NSI to standard charged current processes. 
In any case, one can use atmospheric neutrino data to set much more stringent limits on these couplings, once we use that the $\varepsilon_{\mu \mu}^{f P}$ are well bounded from $\nu_{\mu}$ scattering experiments.

\section{KamLAND and SNO/SK}

The large mixing angle (LMA) oscillation solution of the solar neutrino problem [37], has been confirmed by the anti-neutrino reactor experiment KamLAND. It is a short baseline experiment (matter effects are negligible in the present global allowed region by solar and reactor data) measuring electron anti-neutrinos through a charged current process. We will assume that KamLAND finds an oscillatory signal, so the neutrino parameters $\Delta m_{\text {sol }}^{2}$ and $\theta_{12}$ will be determined with good precision. Let us stress that the neutral current NSI we are discussing in this work does not affect in a significant way the KamLAND observables. On the other hand, the solar neutrino data may be plagued by NSI present in the matter potential (evolution in the Sun and in the Earth) and/or by NSI present in the neutrino neutral current detection. Therefore the consistency of the KamLAND and the solar neutrino data can give us information on NSI that contribute in solar neutrino experiments. In this section, we anticipate the constraints that could be set with three years of KamLAND data.

We will consider solar neutrino data only from SNO and SK (for a discussion on the impact of NSI in the Borexino observables, see Ref. [38]). The main source of neutrinos at the energies relevant for SNO and SK is ${ }^{8} B$ neutrinos. There is also a small contribution due to hep neutrinos (roughly $0.5 \%$ of the total rate if we use the solar standard model fluxes) that we consider as a source of systematic error in the experiments. The reason for using only ${ }^{8} B$ neutrino data is that the resulting bounds on NSI are almost solar model independent. For simplicity, we will use the total number of events measured at these experiments for the daytime and for the nighttime characterized by the total rates and the day-night asymmetries. These data are enough to show the bounds that can be reached by this set of experiments. A more complete analysis should include spectral SK and SNO information.

NSI modify the evolution in matter by the effective parameters $\varepsilon^{\prime V}$ and $\varepsilon^{V}$, described as a function of the fundamental NSI by $\|$

$$
\begin{gathered}
\varepsilon^{V}=\sum_{f=e, u, d}\left(\cos \theta_{23} \varepsilon_{e \mu}^{f V}-\sin \theta_{23} \varepsilon_{e \tau}^{f V}\right) \frac{N_{f}}{N_{e}}, \\
\varepsilon^{\prime V}=\sum_{f=e, u, d}\left(\varepsilon_{e e}^{f V}-\left(\cos ^{2} \theta_{23} \varepsilon_{\mu \mu}^{f V}+\sin ^{2} \theta_{23} \varepsilon_{\tau \tau}^{f V}-\sin 2 \theta_{23} \varepsilon_{\mu \tau}^{f V}\right)\right) \frac{N_{f}}{N_{e}},
\end{gathered}
$$

where $N_{f}$ is the number density of target particles $f$ in matter, and $\varepsilon^{V}=\varepsilon^{L}+\varepsilon^{R}$. We are using the fact that the 3-neutrino evolution in matter can be described in good approximation by an effective 2-neutrino description even for $\varepsilon_{e \tau} \sim 1$, because $G_{F} N_{e} \ll \Delta m_{a t m}^{2} / E_{\nu}$. The neutrino evolution Hamiltonian in the presence of NSI can be found in $[8]$.

Let us discuss the main effect of NSI in the solar neutrino evolution:

- $\varepsilon^{\prime V}$ modifies the survival probability through the change of the effective matter density seen by the neutrinos. If $\varepsilon^{\prime V}$ is negative, the effective density is smaller and the resonance happens at larger energies, changing the recoil energy spectrum and the total rate in SK and SNO. Thus, the evolution of the ${ }^{8} B$ neutrinos in the Sun is sensitive to negative $\varepsilon^{\prime V}$. On the contrary, a positive $\varepsilon^{\prime V}$ increases the effective density and the resonance happens at lower energies, having a flat spectrum for the ${ }^{8} B$ neutrinos and a slightly affected rate. This would imply that there is no bound on positive $\varepsilon^{\prime V}$ from ${ }^{8} B$ neutrinos. However, the large effective density would be also in the Earth and it would make an effect in the day-night difference. The measured day-night asymmetry $\left(A_{D N}=2(N-D) /(N+D)\right.$ where $\mathrm{D}(\mathrm{N})$ are the number of events measured during the daytime (nighttime)) bounds positive $\varepsilon^{\prime V}$.

- $\varepsilon^{V}$, if small, modifies the survival probability through the change of the effective mixing [26]. Roughly speaking, KamLAND depends on $\theta$ while SNO/SK observables depend on the combinations $\theta+\varepsilon^{V}$ and the comparison of the allowed ranges for the mixing angle extracted from KamLAND data and from the SK-SNO data define the $\varepsilon^{V}$ bounds we can get.

We consider the following solar neutrino observables:

\footnotetext{
"In this analysis we have taken $\theta_{13}=0$, which is a good approximation because solar and KamLAND data are weakly sensitive to $\theta_{13}$ below the CHOOZ bound.
} 
SNO Charged Current (CC): NSI appear only in the propagation due to matter effects (only vector couplings contribute),

$$
[C C]=f_{B}\left\langle P_{e e}\left(\varepsilon^{\prime V}, \varepsilon^{V}\right)\right\rangle_{C C}
$$

where

$$
f_{B}=\frac{\Phi_{8}}{\Phi_{8}^{S S M}}
$$

is the ${ }^{8} \mathrm{~B}$ solar neutrino flux normalized to the standard solar model prediction. We denoted $[\mathrm{XX}]$ as the observable XX normalized to the case of no transition $\left([X X]=\frac{X X\left(P_{e e}\right)}{X X\left(P_{e e}=1\right)}\right)$ and \langle\rangle$_{X X}$ indicates the observable XX averaged with the detector response. $P_{e e}$ is the probability that $\nu_{e}$ produced in the sun will arrive as $\nu_{e}$ at the detector.

SNO Neutral Current: NSI appear mainly in the neutrino detection. At low energies the neutrino-deuteron cross section is dominated by the Gamow-Teller transitions, so that the cross section scales as $g_{A}^{2}$, where $g_{A}$ is the coupling of the neutrino current to the axial isovector hadronic current ${ }^{* *}[39,40,41,42,43]$. In the SM, $g_{A}=g_{A}^{u}-g_{A}^{d}=1$ and using that the nuclear corrections to $g_{A}$ are the same when the NSI are added we obtain:

$$
[N C] \sim f_{B}\left(1+2 \varepsilon^{A}\right)
$$

where

$$
\varepsilon^{A} \sim \sum_{\alpha=e, \mu, \tau}\left\langle P_{e \alpha}\right\rangle_{N C}\left(\varepsilon_{\alpha \alpha}^{u A}-\varepsilon_{\alpha \alpha}^{d A}\right)
$$

Thus, this cross section is mainly sensitive to the axial part of the NSI and contains complementary information to the oscillation probabilities that depend on the vector part of the NSI. Notice that in the case of absence of axial NSI, the NC detection is blind to oscillations $\left(\sum_{\alpha=e, \mu, \tau} P_{e \alpha}=1\right)$ and determine the total ${ }^{8} \mathrm{~B}$ flux even if neutrinos oscillate [44].

SNO-SK Elastic Scattering: NSI appear in the neutrino evolution and also in the neutrino detection [38].

$$
[E S]=f_{B}\left[r_{e}\left\langle P_{e e}\left(\varepsilon^{\prime V}, \varepsilon^{V}\right)\right\rangle_{E S}+0.157 r_{a}\left(1-\left\langle P_{e e}\left(\varepsilon^{\prime V}, \varepsilon^{V}\right)\right\rangle_{E S}\right)\right]
$$

where

$$
\begin{aligned}
r_{e}= & \frac{1}{\sigma_{\nu_{e} e}\left(g_{L}, g_{R}\right)} \sigma_{\nu_{e} e}\left(g_{L}+\varepsilon_{e e}^{e L}, g_{R}+\varepsilon_{e e}^{e R}, \sum_{\alpha \neq e}\left|\varepsilon_{\alpha e}^{e P}\right|^{2}\right), \\
r_{a} & =\frac{\cos ^{2} \theta_{23}}{\sigma_{\nu_{\mu} e}\left(g_{L}, g_{R}\right)} \sigma_{\nu_{\mu} e}\left(g_{L}+\varepsilon_{\mu \mu}^{e L}, g_{R}+\varepsilon_{\mu \mu}^{e R}, \sum_{\alpha \neq \mu}\left|\varepsilon_{\alpha \mu}^{e P}\right|^{2}\right) \\
& +\frac{\sin ^{2} \theta_{23}}{\sigma_{\nu_{\tau} e}\left(g_{L}, g_{R}\right)} \sigma_{\nu_{\tau} e}\left(g_{L}+\varepsilon_{\tau \tau}^{e L}, g_{R}+\varepsilon_{\tau \tau}^{e R}, \sum_{\alpha \neq \tau}\left|\varepsilon_{\alpha \tau}^{e P}\right|^{2}\right) .
\end{aligned}
$$

$\sigma_{\nu_{e} e}\left(g_{L}+\varepsilon_{e e}^{e L} \ldots\right)$ is given in eq. (4); the $\sigma_{\nu_{\mu} e}\left(g_{L}+\varepsilon_{\mu \mu}^{e L} \ldots\right)$ and $\sigma_{\nu_{\tau} e}\left(g_{L}+\varepsilon_{\tau \tau}^{e L} \ldots\right)$ are the obvious modifications of this equation. In the following, we refer to [ES] as the averaged value from SK and SNO electron scattering measurements.

Regarding the data used in the present analysis, we assume that in the next three years:

- KamLAND will find a clear signal of oscillations (total rate and energy distortion). From KamLAND, we will be able to know the ranges of the solar oscillation parameters, $\Delta m_{\text {sol }}^{2}$ and $\theta_{12}$ with good precision. Assuming that KamLAND confirm the present best fit point to the solar neutrino data, we expect $\left\langle P_{e e}\left(\varepsilon^{\prime V}=0, \varepsilon^{V}=0\right)\right\rangle=$ $0.32(1 \pm 0.07)[45]$.

- We assume a moderate improvement on the SNO and SK measurements and that the NC is measured independently from the CC in the second and the third SNO phases [46]. The central values correspond to the present central measurements : $[\mathrm{CC}]=0.35(1 \pm 0.05),[\mathrm{NC}]=1.01(1 \pm 0.08)$ and $[\mathrm{ES}]=0.47(1 \pm 0.03)$ (the present measurements and errors are $[\mathrm{CC}]=0.349(1 \pm 0.057)[2],[\mathrm{NC}]=1.008(1 \pm 0.125)[2],[\mathrm{ES}]=0.465(1 \pm 0.032)[47])$.

- We use the day-night asymmetry measured at Super-Kamiokande $A_{D N}=0.021 \pm 0.024$ [47], consistent with the first SNO measurement $A_{D N}=0.07 \pm 0.05$ [48].

**We are grateful to José Bernabéu for an enlightening discussion about this point. 
- In the calculations, we use $0.4 \leq \cos ^{2} \theta_{23} \leq 0.6$, the expected $90 \%$ CL allowed range from MINOS [49].

Finally, the results presented in this section have been obtained solving numerically the evolution in the Sun and in the Earth using the electron and neutron number densities from the standard solar model [50] and the preliminary reference earth model [51]. Details on the KamLAND simulation used in this analysis can be found in Ref. [45]. CC and NC cross sections were obtained from Ref. [41]. ES was computed including radiative corrections [52].

We present bounds on a given four-fermion operator, assuming that all other four-fermion operators are zero. Let us illustrate how the bounds come out for a particular NSI, namely $\left(\bar{e} \gamma^{\rho} L e\right)\left(\bar{\nu}_{e} \gamma_{\rho} L \nu_{e}\right)$. If we consider only this operator, eqs. (63)-(67) simplify to:

$$
\begin{gathered}
{[C C]=f_{B}\left\langle P_{e e}\left(\varepsilon_{e e}^{e L}\right)\right\rangle_{C C},} \\
{[N C]=f_{B}} \\
{[E S]=f_{B}\left(r_{e}\left\langle P_{e e}\left(\varepsilon_{e e}^{e L}\right)\right\rangle_{E S}+0.157\left(1-\left\langle P_{e e}\left(\varepsilon_{e e}^{e L}\right)\right\rangle_{E S}\right)\right) .}
\end{gathered}
$$

In that case, the $\mathrm{NC}$ value is a measurement of the ${ }^{8} \mathrm{~B}$ flux, $f_{B}=1.01(1 \pm 0.08)$. This result for $f_{B}$ combined with eq. (70) gives $\left\langle P_{e e}\left(\varepsilon_{e e}^{e L}\right)\right\rangle_{C C}=0.33(1 \pm 0.09)$. By inspection, if we compare this averaged rate with the result from the KamLAND allowed region, $\left\langle P_{e e}\left(\varepsilon^{\prime V}=0, \varepsilon^{V}=0\right)\right\rangle=0.32(1 \pm 0.07)$, we can conclude that $\varepsilon_{e e}^{e L}$ must be constrained in the range where matter effects from the NSI are small. Using this conclusion in eq. (72), $\varepsilon_{e e}^{e L}$ is further constrained by the ES dependence on $r_{e}$. Solving numerically the set of eqs. (70)-(72) as a function of $f_{B}$ and $\varepsilon_{e e}^{e L}$ and using the allowed range of parameters $\left(\Delta m_{s o l}^{2}, \theta_{12}\right)$ from KamLAND, we get the 90\%CL bound :

$$
-0.08<\varepsilon_{e e}^{e L}<0.10 .
$$

More precisely, given the solar observables considered, we computed $\chi_{s o l}^{2}$ as a function of the NSI parameter $\varepsilon_{\alpha \beta}^{f P}$, the oscillation parameters $\Delta m_{s o l}^{2}, \theta_{12}$ and $\theta_{23}$, and the ${ }^{8} \mathrm{~B}$ flux normalization, $f_{B}$. We add the $\chi^{2}$ of the KamLAND analysis, a function of $\Delta m_{\text {sol }}^{2}$ and $\theta_{12}$, to the $\chi_{\text {sol }}^{2}$. Next, we find the marginalized $\chi^{2}\left(\varepsilon_{\alpha \beta}^{f P}\right)$ by minimizing the total $\chi^{2}$, $\chi_{\text {total }}^{2}=\chi_{\text {sol }}^{2}+\chi_{\text {KamLAND }}^{2}$, respect to the oscillation parameters and the ${ }^{8} \mathrm{~B}$ flux normalization for each $\varepsilon_{\alpha \beta}^{f P}$. Finally, we get the bounds on the NSI parameter by comparing the function $\Delta \chi^{2}\left(\varepsilon_{\alpha \beta}^{f P}\right)=\chi^{2}\left(\varepsilon_{\alpha \beta}^{f P}\right)-\chi_{\min }^{2}$ and the statistical $\chi^{2}$ distribution with 1 dof $\left(\chi_{\min }^{2}\right.$ is the minimum of $\chi^{2}$ (total) in the full space of parameters). For the different operators (one by one) that appear in the solar observables, we get at $90 \% \mathrm{CL}$ :

$$
\begin{gathered}
-0.2<\varepsilon_{\alpha \alpha}^{e L}<0.3 \\
-0.3<\varepsilon_{e e}^{e R}<0.5 \\
-0.9<\varepsilon_{\alpha \alpha}^{e R}<0.3 \\
-0.25<\varepsilon_{e e}^{u L}<0.2 \\
-0.3<\varepsilon_{\alpha \alpha}^{u L}<0.25 \\
-0.2<\varepsilon_{e e}^{u R}<0.25 \\
-0.25<\varepsilon_{\alpha \alpha}^{u R}<0.3 \\
-0.2<\varepsilon_{e e}^{d L}<0.25 \\
-0.25<\varepsilon_{\alpha \alpha}^{d L}<0.3 \\
-0.25<\varepsilon_{e e}^{d R}<0.2 \\
-0.3<\varepsilon_{\alpha \alpha}^{d R}<0.25
\end{gathered}
$$

where $\alpha=\mu, \tau$ and

$$
-0.2<\varepsilon_{\alpha \beta}^{f P}<0.3
$$

where $f=e, u, d, P=L, R$, and $\alpha, \beta=e, \mu, \tau$ with $\alpha \neq \beta$.

\section{$5 \quad \nu$ Factory}

In this section, we estimate the sensitivity to NSI of $\nu e$ scattering and $\nu$-DIS at the near detector of a neutrino factory. We outline in the next paragraphs the order of magnitude of the limits the near detector could set on $\varepsilon_{\alpha \alpha}(\alpha \neq \tau)$, and $\varepsilon_{\alpha \beta}(\alpha \neq \beta)$. A more careful analysis follows in subsections 5.1 and 5.2, based on the $\nu$-DIS chapter of the 
ECFA-CERN Neutrino Factory Study [53]. Mangano et al. [53] discuss various measurements from which $\sin ^{2} \theta_{W}$ could be extracted, with their potential errors. Finally, in section 5.3, we briefly review potential nufactory bounds on new charged current neutrino interactions $[16,53]$.

For flavour diagonal NSI involving $\nu_{e}$ and $\nu_{\mu}, \sin ^{2} \theta_{W}$ measurements at a near detector of a neutrino factory should be more sensitive than oscillation probabilities measured at the far detector. $\varepsilon_{\alpha \alpha}$ will interfere with the SM amplitude for $\nu_{\alpha} f \rightarrow \nu_{\alpha} f{ }^{\dagger \dagger}$ so the diagonal NSI contribute linearly to scattering. They also contribute linearly to the oscillation probability at the far detector, via their MSW contribution to the neutrino mass matrix. But there should be more events at the near detector, because the near beam is narrow, so a well-instrumented detector, of area larger than the beam, can be built at reasonable cost. This suggests that the statistical and systematic errors on $\varepsilon_{e e}$ and $\varepsilon_{\mu \mu}$ would be smaller at a near detector.

The situation is less clear for flavour changing $\varepsilon_{\alpha \beta}(\alpha \neq \beta)$ which contribute quadratically to $\sin ^{2} \theta_{W}$. Neglecting for the moment systematic errors, one can estimate that the near detector would be sensitive to NSI such that $N_{N S I}^{n} \gtrsim \sqrt{N_{S M}^{n}}$. The number of events in the near detector due to NSI is $N_{N S I}^{n} \propto \varepsilon^{2} N_{S M}^{n}$, where $N_{S M}^{n}$ is the number of SM $\nu$ scattering events. In the analysis of [53], $N_{S M}^{n} \sim 10^{8}$ was taken, so $\varepsilon_{\alpha \beta} \gtrsim .01$ might be seen. At the far detector $[10,14,15], \varepsilon_{\alpha \beta} \mathrm{s}$ ( with $\alpha \neq \beta$ ) appear linearly in the oscillation probability, because they interfere with the SM oscillation amplitude. NSI could disrupt the measurement of oscillation parameters at the far detector if $N_{N S I}^{f} \gtrsim N_{o s c}^{f}$, where $N_{o s c}^{f}$ is the number of events at the far detector due to oscillations. So $\varepsilon_{\alpha \beta} \gtrsim \sin \theta_{13} \operatorname{could}$ interfere with the measurement of $\sin \theta_{13}$ at the far detector [16]. A recent analysis [54] suggests that a neutrino factory can realistically measure $\sin \theta_{13} \gtrsim .01$. We find that the near detector would be sensitive to $\varepsilon_{\alpha \beta} \gtrsim$ a few $\times .01$, so the sensitivities of the near and far detector to flavour-changing NSI are similar.

\subsection{Measuring $\sin ^{2} \theta_{W}$ leptonically}

The weak mixing angle could be measured leptonically in the scattering of neutrinos off electrons in the target. The error is smaller using the $\bar{\nu}_{\mu}, \nu_{e}$ from the $\mu^{+}$beam; $\sin ^{2} \theta_{W}$ can be determined from

$$
\sigma_{\nu f a c t}=\sigma\left(\bar{\nu}_{\mu} e \rightarrow \bar{\nu} e\right)+\sigma\left(\nu_{e} e \rightarrow \nu e\right)
$$

with a statistical error of order $\Delta \sin ^{2} \theta_{W}=2 \times 10^{-4}$ [53]. To estimate "90\% C.L." limits on NSI, we multiply by 1.6, so use $\Delta \sin ^{2} \theta_{W}=3 \times 10^{-4}$. The second of these two cross-sections, augmented by the contributions of NSI, is given in eq. (4), and the first is

$$
\sigma\left(\bar{\nu}_{\mu} e \rightarrow \bar{\nu} e\right)=\frac{2 G_{F}^{2} m_{e} E_{\nu}}{\pi}\left[\frac{1}{3}\left(g_{L}^{e}+\varepsilon_{\mu \mu}^{e L}\right)^{2}+\frac{1}{3} \sum_{\alpha \neq \mu}\left|\varepsilon_{\mu \alpha}^{e L}\right|^{2}+\left(g_{R}^{e}+\varepsilon_{\mu \mu}^{e R}\right)^{2}+\sum_{\alpha \neq \mu}\left|\varepsilon_{\mu \alpha}^{e R}\right|^{2}\right]
$$

Requiring $\left(\sigma_{\nu f a c t}-\sigma_{S M}\right) \lesssim \Delta s_{W}^{2} \times \partial \sigma_{S M} / \partial s_{W}^{2}$ gives

$$
\begin{aligned}
& \frac{2}{3} g_{L}^{e} \varepsilon_{\mu \mu}^{e L}+\frac{1}{3} \sum_{\alpha}\left|\varepsilon_{\mu \alpha}^{e L}\right|^{2}+2 g_{R}^{e} \varepsilon_{\mu \mu}^{e R}+\sum_{\alpha}\left|\varepsilon_{\mu \alpha}^{e R}\right|^{2} \\
& \quad+2\left(1+g_{L}^{e}\right) \varepsilon_{e e}^{e L}+\sum_{\alpha}\left|\varepsilon_{\alpha e}^{e L}\right|^{2}+\frac{2}{3} g_{R}^{e} \varepsilon_{e e}^{e R}+\frac{1}{3} \sum_{\alpha}\left|\varepsilon_{\alpha e}^{e R}\right|^{2}<6 \times 10^{-4},
\end{aligned}
$$

so the flavour-changing NSI satisfy

$$
\begin{array}{ll}
\left|\varepsilon_{\mu \tau}^{e L}\right|<.04 & \left|\varepsilon_{\mu \tau}^{e R}\right|<.02 \\
\left|\varepsilon_{\tau e}^{e L}\right|<.02 & \left|\varepsilon_{\tau e}^{e R}\right|<.04 .
\end{array} .
$$

(We neglect $\varepsilon_{\mu e}^{e P}$ because it is more strongly constrained by $\mu \rightarrow 3 e$ - see section 3.2.) The flavour diagonal $\varepsilon$ s satisfy

$$
\left|\varepsilon_{\mu \mu}^{e L}\right|<0.003 \quad,\left|\varepsilon_{\mu \mu}^{e R}\right|<0.001 \quad,\left|\varepsilon_{e e}^{e L}\right|<0.0004 \quad,\left|\varepsilon_{e e}^{e R}\right|<0.004
$$

assuming no cancellations in equation (88).

\subsection{Measuring $\sin ^{2} \theta_{W}$ in DIS}

In neutrino Deep Inelastic Scattering (DIS), the NC events due to incident $\nu_{e}$ and $\bar{\nu}_{\mu}$ cannot be separated. So the Paschos-Wolfenstein ratio:

$$
R_{P W}=\frac{\sigma_{N C}\left(\nu_{\mu}\right)-\sigma_{N C}\left(\bar{\nu}_{\mu}\right)}{\sigma_{C C}\left(\nu_{\mu}\right)-\sigma_{C C}\left(\bar{\nu}_{\mu}\right)}
$$

\footnotetext{
${ }^{\dagger} \alpha \neq \tau$ here
} 
is not available, and $s_{W}^{2}$ cannot be so elegantly disentangled from parton distributions. It was conservatively estimated in [53] that a neutrino factory could measure $s_{W}^{2}$ to one part in $10^{3}$, via a ratio of the form

$$
\begin{aligned}
R_{\mu}^{-} & =\frac{\sigma_{N C}\left(\nu_{\mu}\right)+\sigma_{N C}\left(\bar{\nu}_{e}\right)}{\sigma_{C C}\left(\nu_{\mu}\right)+\sigma_{C C}\left(\bar{\nu}_{e}\right)} \\
& =\frac{\left(\tilde{g}_{L \mu}\right)^{2}+\left(\tilde{g}_{R e}\right)^{2}+r\left[\left(\tilde{g}_{R \mu}\right)^{2}+\left(\tilde{g}_{L e}\right)^{2}\right]}{1+r}
\end{aligned}
$$

where $r \sim 0.5$ is defined in eq.(26), and the $\tilde{g}$ with lepton subscripts are as defined in equations (11),(12), replacing $e \rightarrow \mu$ as required.

A similar ratio $R^{+}$can be defined for the beam produced in the decay of a $\mu^{+}$:

$$
\begin{aligned}
R_{\mu}^{+} & =\frac{\sigma_{N C}\left(\bar{\nu}_{\mu}\right)+\sigma_{N C}\left(\nu_{e}\right)}{\sigma_{C C}\left(\bar{\nu}_{\mu}\right)+\sigma_{C C}\left(\nu_{e}\right)} \\
& =\frac{\left(\tilde{g}_{R \mu}\right)^{2}+\left(\tilde{g}_{L e}\right)^{2}+r\left[\left(\tilde{g}_{L \mu}\right)^{2}+\left(\tilde{g}_{R e}\right)^{2}\right]}{1+r}
\end{aligned}
$$

$R^{+}$and $R^{-}$can be measured with equal sensitivity, so $s_{W}^{2}$ can be independently determined from both. The NSI appear in one or the other not multiplied by $r$, so we can set bounds of order $\Delta\left(\tilde{g}_{P \mu, e}\right)^{2} \lesssim \partial R^{ \pm} / \partial s_{W}^{2} \times 10^{-3} \sim .0005$, for instance

$$
\frac{2 g_{L}^{u} \varepsilon_{\mu \mu}^{u L}}{1+r}<5 \times 10^{-4}
$$

or equivalently

$$
\left|\varepsilon_{\mu \mu}^{u L}\right|<1 \times 10^{-3}
$$

and assuming no cancellations among the terms in $\tilde{g}_{P \ell}$ (eqs.(11),(12)) :

$$
\begin{aligned}
& \left|\varepsilon_{\mu \mu}^{u L}\right|,\left|\varepsilon_{e e}^{u L}\right|<1 \times 10^{-3}, \quad\left|\varepsilon_{\mu \mu}^{d L}\right|,\left|\varepsilon_{e e}^{d L}\right|<9 \times 10^{-4} \\
& \left|\varepsilon_{\mu \mu}^{u R}\right|,\left|\varepsilon_{e e}^{u R}\right|<2 \times 10^{-3}, \quad\left|\varepsilon_{\mu \mu}^{d R}\right|,\left|\varepsilon_{e e}^{d R}\right|<5 \times 10^{-3} \\
& \left|\varepsilon_{\tau \mu}^{q L}\right|,\left|\varepsilon_{\tau e}^{q L}\right|,\left|\varepsilon_{\tau \mu}^{q R}\right|,\left|\varepsilon_{\tau e}^{q R}\right|<3 \times 10^{-2} .
\end{aligned}
$$

\subsection{Charged current interactions}

Non standard neutrino interactions of the form given in equation (2) could contribute at one loop to muon decay or charged current $\nu$ scattering off quarks in the near detector. This would occur via the external dressing of the four fermion operator with a $W$ loop, as discussed in section 3.2. For instance, exchanging a $W$ between the $\nu_{\mu}$ and $e$ legs of $\varepsilon\left(\bar{\nu}_{\tau} \gamma^{\rho} \nu_{\mu}\right)\left(\bar{e} \gamma_{\rho} L e\right)$ would generate the operator $c \varepsilon\left(\bar{\nu}_{\tau} \gamma^{\rho} \nu_{e}\right)\left(\bar{e} \gamma_{\rho} L \mu\right)$. This would produce a $\nu_{\tau}$ from $\mu$ decay, which could turn into a $\tau$ in CC scattering off quarks. The sensitivity of the near detector to operators of the form $2 \sqrt{2} G_{F} \eta\left(\bar{e} \gamma_{\rho} \mu\right)\left(\bar{\nu}_{\beta} \gamma^{\rho} \nu_{\alpha}\right)$ was estimated in [53] (see also [12, 16, 55]) to be

$$
\eta \lesssim 10^{-4} \sqrt{N} \times \sqrt{\frac{\rho_{\text {det }}}{100 g / c m^{2}}} \sqrt{\frac{\text { number of } \mu \text { decays }}{10^{20}}}
$$

where $N$ is the number of $\tau$ events required in the detector for a signal. $N \sim 10$ was taken in [53]. For $c \sim .002$, this is a weaker bound on $\varepsilon_{e \tau}^{e P}, \varepsilon_{\mu \tau}^{e P}$ than from measuring $\sin ^{2} \theta_{W}$. However, in the opposite limit of $c \sim 1$ (for instance if the NSI are induced by dimension 6 operators), it is clear that flavour changing NSI are more readily detected via CC interactions than $\mathrm{NC}$ interactions.

\section{Summary}

We have considered non-standard interactions of neutrinos with first generation leptons and quarks, parametrised as

$$
\mathcal{L}_{\text {eff }}^{N S I}=-\varepsilon_{\alpha \beta}^{f P} 2 \sqrt{2} G_{F}\left(\bar{\nu}_{\alpha} \gamma_{\rho} L \nu_{\beta}\right)\left(\bar{f} \gamma^{\rho} P f\right) .
$$

We have taken a phenomenological approach, assuming that the new physics which induces these non-standard neutral current operators does not generate the $S U(2)$ related charged lepton operators at tree level. This could be the case if the operators in (98) are of dimension eight or larger. Then the tight bounds on charged lepton NSI do not apply, so we have assembled present and future constraints on such operators from purely neutrino processes. 
We point out though, that even if only neutrino neutral current NSI are present at tree level, they will necessarily induce the related vertices with charged leptons at one loop, via $W$ exchange. Moreover, radiative corrections involving these neutrino NSI could affect a variety of precision observables. Thus we have also set bounds on the strength of the NSI in (98) from their one loop effects, which in some cases are more stringent than the tree level ones.

Our results are summarized in tables 2 and 3. We list the limit on $\varepsilon_{\alpha \beta}^{f P}$ that an experiment would set if only one NSI operator was present. The limits that arise if cancellations are allowed among the operators are presented in the body of the paper.

Tree level bounds arise from low energy neutrino scattering experiments. We have collected present constraints, including the recent $\mathrm{NuTeV}$ data, and estimated future limits attainable at the near detector of a neutrino factory from measuring $\sin ^{2} \theta_{W}$ (both, leptonically and in neutrino DIS). We found that these experiments are more sensitive to $\varepsilon_{e e}^{f P}$ and $\varepsilon_{\mu \mu}^{f P}$ than the $\nu$ factory far detector. They also provide the best bounds on flavour changing interactions involving $\nu_{\tau}$.

One loop bounds on lepton flavour violating operators are very stringent for $\varepsilon_{e \mu}^{f P}$. The present experimental limits are so strong, that even with the loop suppression, the $\varepsilon_{e \mu}^{f P}$ are constrained to be $\mathcal{O}\left(10^{-3}\right)$. The analogous bounds on $\varepsilon_{\tau \alpha}^{f P}(\alpha=e, \mu)$ from several tau decays are just order one, although the experimental limits on some of these decays could improve, in which case the relevant bound can be rescaled. Regarding flavour diagonal NSI, one loop bounds on $\varepsilon_{\tau \tau}^{e P}$ from the precise measurement of $g_{A}^{e}$ at LEP are of the same order as the tree level limits from $e^{+} e^{-} \rightarrow \gamma \nu \bar{\nu}$, while the limits on $\varepsilon_{\tau \tau}^{q P}$ from the invisible $Z$ width are the only constraints from laboratory data.

Finally, we have estimated future bounds from comparing KamLAND and solar neutrino data. These could constrain $\varepsilon_{\tau \tau}^{q P}$ at the level of $\mathcal{O}(0.3)$ (which is roughly one order of magnitude improvement of the present limits), and set bounds on $\varepsilon_{\tau \tau}^{e P}$ which are comparable to those from LEP.

We hoped to show that long-baseline experiments at a neutrino factory would indeed measure oscillation parameters, since NSI would be seen first in other experiments, like short baseline, high intensity, precision neutrino scattering experiments. To what degree have we succeeded?

Suppose that no evidence for NSI is found; does this mean such interactions can be ignored in long baseline oscillation experiments? That is, if the near detector of a neutrino factory sees no NSI, will the far detector measure oscillation parameters? The answer we find seems to be that the NSI cannot quite be ignored. The three problematic $\varepsilon \mathrm{s}$ are $\varepsilon_{\tau \tau}, \varepsilon_{\tau \mu}$ and $\varepsilon_{\tau e}$.

The Mangano et al. [53] analysis suggests that the near detector would be sensitive to $\varepsilon_{\tau \mu}, \varepsilon_{\tau e} \gtrsim .02$, and the Freund et al. analysis [54] says the far detector could see $\sin \theta_{13} \lesssim .01$. We would like $\varepsilon_{\alpha \beta}<\sin \theta_{13}$ to be sure that the $\varepsilon$ s do not confuse the determination of $\sin \theta_{13}$. Can these two analyses be compared? Freund et al. use more events, but the error in the near detector analysis of [53] is systematic, so it is not clear how the errors scale if we increase the number of events. The error on the DIS determination of $\sin ^{2} \theta_{W}$ could be significantly smaller than estimated in [53], because the source of error is the parton distribution functions, and the near detector will measure these [56]. So the near detector might indeed be able to constrain $\varepsilon_{\tau \mu}^{q P}, \varepsilon_{\tau e}^{q P}<.01$. Also, $\varepsilon_{\tau \mu}^{q} \gtrsim .01$ could possibly be seen at ICARUS/OPERA [11], where the neutrino beam is largely $\nu_{\mu}$ produced in pion decay, and the detector should be able to identify $\tau \mathrm{s}$.

$\varepsilon_{\tau \mu}^{e P}, \varepsilon_{\tau e}^{e P}$ remain a problem, but could perhaps be disentangled from $\theta_{13}$ at a neutrino factory by using the beam spectrum, as discussed in [17].

\section{Acknowledgments}

We thank Anna Rossi for stimulating our interest in this physics. We also thank Jose Bernabéu, Paolo Gambino, Michele Maltoni and Antonio Pich for discussions, and Cecilia Lunardini, Alexander Friedland and Jose Valle for comments on the manuscript. This work was partially supported by the Spanish MCyT grants BFM2002-00345, BFM2002-00568 and FPA2001-3031, by the TMR network contract HPRN-CT-2000-00148 of the European Union and by the Generalitat Valenciana grants CTIDIB/2002/24 and GV01-94. CPG acknowledges support from NSF grant No. PHY-0070928. 
Table 2: Flavour conserving four fermion vertices involving two neutrinos and two first generation fermions $(\bar{e} e, \bar{d} d$ or $\bar{u} u)$, the best current and the best future $90 \% \mathrm{CL}$ limits that can be set on the coefficients $2 \sqrt{2} G_{F} \varepsilon$ of the four fermion vertices. See eq. (2) for the definition of $\varepsilon$. The limits from processes marked with an asterisk, ${ }^{*}$, arise at one loop and are inversely proportional to $\log \left(\Lambda / m_{W}\right)$. We have assumed $\log \left(\Lambda / m_{W}\right)>1$ (see section 3.2).

\begin{tabular}{|c|c|c|}
\hline vertex & current limits & future limit \\
\hline$\left(\bar{e} \gamma^{\rho} P e\right)\left(\bar{\nu}_{\tau} \gamma_{\rho} L \nu_{\tau}\right)$ & $\begin{array}{c}\left|\varepsilon_{\tau \tau}^{e P}\right|<0.5 \\
\left.\left(g_{A}^{e} @ \mathrm{LEP}\right)^{*}\right)\end{array}$ & $\begin{array}{c}-0.2<\varepsilon_{\tau \tau}^{e L}<0.3 \\
-0.9<\varepsilon_{\tau \tau}^{e R}<0.3 \\
\text { KamLAND and SNO } / \mathrm{SK}\end{array}$ \\
\hline$\left(\bar{u} \gamma^{\rho} P u\right)\left(\bar{\nu}_{\tau} \gamma_{\rho} L \nu_{\tau}\right)$ & $\begin{array}{c}\left|\varepsilon_{\tau \tau}^{u L}\right|<1.4 \\
\left|\varepsilon_{\tau \tau}^{u R}\right|<3 \\
\left(\Gamma_{i n v}\right)^{*)}\end{array}$ & $\begin{array}{c}-0.3<\varepsilon_{\tau \tau}^{u L}<0.25 \\
-0.25<\varepsilon_{\tau \tau}^{u R}<0.3 \\
\text { KamLAND and SNO/SK }\end{array}$ \\
\hline$\left(\bar{d} \gamma^{\rho} L d\right)\left(\bar{\nu}_{\tau} \gamma_{\rho} L \nu_{\tau}\right)$ & $\begin{array}{c}\left|\varepsilon_{\tau \tau}^{d L}\right|<1.1 \\
\left|\varepsilon_{\tau \tau}^{d R}\right|<6 \\
\left(\Gamma_{i n v}\right)^{*)}\end{array}$ & $\begin{array}{c}-0.25<\varepsilon_{\tau \tau}^{d L}<0.3 \\
-0.3<\varepsilon_{\tau \tau}^{d R}<0.25 \\
\text { KamLAND and SNO/SK }\end{array}$ \\
\hline$\left(\bar{e} \gamma^{\rho} P e\right)\left(\bar{\nu}_{\mu} \gamma_{\rho} L \nu_{\mu}\right)$ & $\begin{array}{l}\left|\varepsilon_{\mu \mu}^{e P}\right|<0.03 \\
\text { CHARM II }\end{array}$ & $\begin{array}{c}\left|\varepsilon_{\mu \mu}^{e L}\right|<0.003 \\
\left|\varepsilon_{\mu \mu}^{e R}\right|<0.001 \\
\text { leptonic } s_{W}^{2} \text { at nufact }\end{array}$ \\
\hline$\left(\bar{u} \gamma^{\rho} P u\right)\left(\bar{\nu}_{\mu} \gamma_{\rho} L \nu_{\mu}\right)$ & $\begin{array}{c}\left|\varepsilon_{\mu \mu}^{u L}\right|<0.003 \\
-0.008<\varepsilon_{\mu \mu}^{u R}<0.003 \\
\mathrm{NuTeV}\end{array}$ & $\begin{array}{c}\left|\varepsilon_{\mu \mu}^{u L}\right|<0.001 \\
\left|\varepsilon_{\mu \mu}^{u R}\right|<0.002 \\
s_{W}^{2} \text { in DIS at nufact }\end{array}$ \\
\hline$\left(\bar{d} \gamma^{\rho} P d\right)\left(\bar{\nu}_{\mu} \gamma_{\rho} L \nu_{\mu}\right)$ & $\begin{array}{c}\left|\varepsilon_{\mu \mu}^{d L}\right|<0.003 \\
-0.008<\varepsilon_{\mu \mu}^{d R}<0.015 \\
\mathrm{NuTeV}\end{array}$ & $\begin{array}{c}\left|\varepsilon_{\mu \mu}^{d L}\right|<0.0009 \\
\left|\varepsilon_{\mu \mu}^{d R}\right|<0.005 \\
s_{W}^{2} \text { in DIS at nufact }\end{array}$ \\
\hline$\left(\bar{e} \gamma^{\rho} P e\right)\left(\bar{\nu}_{e} \gamma_{\rho} L \nu_{e}\right)$ & $\begin{array}{c}-0.07<\varepsilon_{e e}^{e L}<0.1 \\
-1<\varepsilon_{e e}^{e R}<0.5 \\
\text { LSND }\end{array}$ & $\begin{array}{c}\left|\varepsilon_{e e}^{e L}\right|<0.0004 \\
\left|\varepsilon_{e e}^{e R}\right|<0.004 \\
\text { leptonic } s_{W}^{2} \text { at nufact }\end{array}$ \\
\hline$\left(\bar{u} \gamma^{\rho} P u\right)\left(\bar{\nu}_{e} \gamma_{\rho} L \nu_{e}\right)$ & $\begin{array}{c}-1<\varepsilon_{e e}^{u L}<0.3 \\
-0.4<\varepsilon_{e e}^{u R}<0.7 \\
\text { CHARM }\end{array}$ & $\begin{array}{c}\left|\varepsilon_{e e}^{u L}\right|<0.001 \\
\left|\varepsilon_{e e}^{u R}\right|<0.002 \\
s_{W}^{2} \text { in DIS at nufact }\end{array}$ \\
\hline$\left(\bar{d} \gamma^{\rho} P d\right)\left(\bar{\nu}_{e} \gamma_{\rho} L \nu_{e}\right)$ & $\begin{array}{c}-0.3<\varepsilon_{e e}^{d L}<0.3 \\
-0.6<\varepsilon_{e e}^{d R}<0.5 \\
\text { CHARM }\end{array}$ & $\begin{array}{c}\left|\varepsilon_{e e}^{d L}\right|<0.0009 \\
\left|\varepsilon_{e e}^{d R}\right|<0.005 \\
s_{W}^{2} \text { in DIS at nufact }\end{array}$ \\
\hline
\end{tabular}


Table 3: Flavour changing four fermion vertices involving two neutrinos and two first generation fermions $(\bar{e} e, \bar{d} d$ or $\bar{u} u)$, the best current, and the best future $90 \% \mathrm{CL}$ limits that can be set on their coefficients $2 \sqrt{2} G_{F} \varepsilon$. See eq. (2) for the definition of $\varepsilon$. The limits from processes marked with an asterisk, ${ }^{*)}$, arise at one loop and are inversely proportional to $\log \left(\Lambda / m_{W}\right)$. We have assumed $\log \left(\Lambda / m_{W}\right)>1$ (see section 3.2).

\begin{tabular}{|c|c|c|}
\hline vertex & current limits & future limit \\
\hline$\left(\bar{e} \gamma^{\rho} P e\right)\left(\bar{\nu}_{\tau} \gamma_{\rho} L \nu_{\mu}\right)$ & $\begin{array}{c}\left|\varepsilon_{\tau \mu}^{e P}\right|<1.2 \\
(\tau \rightarrow \mu \bar{e} e)^{*)} \\
\left|\varepsilon_{\tau \mu}^{e P}\right|<0.1 \\
\text { CHARM II }\end{array}$ & $\begin{array}{c}\left|\varepsilon_{\tau \mu}^{e L}\right|<0.04,\left|\varepsilon_{\tau \mu}^{e R}\right|<0.02 \\
\text { leptonic } s_{W}^{2} \text { at nufact }\end{array}$ \\
\hline$\left(\bar{u} \gamma^{\rho} P u\right)\left(\bar{\nu}_{\tau} \gamma_{\rho} L \nu_{\mu}\right)$ & $\begin{array}{c}\left|\varepsilon_{\tau \mu}^{u P}\right|<2.8 \\
\left.(\tau \rightarrow \mu \rho)^{*}\right) \\
\left|\varepsilon_{\tau \mu}^{u P}\right|<0.05 \\
\mathrm{NuTeV} \\
\end{array}$ & $\begin{array}{c}\left|\varepsilon_{\tau \mu}^{u P}\right|<0.03 \\
s_{W}^{2} \text { in DIS at nufact }\end{array}$ \\
\hline$\left(\bar{d} \gamma^{\rho} P d\right)\left(\bar{\nu}_{\tau} \gamma_{\rho} L \nu_{\mu}\right)$ & $\begin{array}{c}\left|\varepsilon_{\tau \mu}^{d P}\right|<2.8 \\
(\tau \rightarrow \mu \rho)^{*)} \\
\left|\varepsilon_{\tau \mu}^{d P}\right|<0.05 \\
\mathrm{NuTeV}\end{array}$ & $\begin{array}{c} \\
\left|\varepsilon_{\tau \mu}^{d P}\right|<0.03 \\
s_{W}^{2} \text { in DIS at nufact }\end{array}$ \\
\hline$\left(\bar{e} \gamma^{\rho} P e\right)\left(\bar{\nu}_{\mu} \gamma_{\rho} L \nu_{e}\right)$ & $\begin{array}{c}\left|\varepsilon_{\mu e}^{e P}\right|<5 \times 10^{-4} \\
(\mu \rightarrow 3 e)^{*)}\end{array}$ & \\
\hline$\left(\bar{u} \gamma^{\rho} P u\right)\left(\bar{\nu}_{\mu} \gamma_{\rho} L \nu_{e}\right)$ & $\begin{array}{c}\left|\varepsilon_{\mu e}^{u P}\right|<7.7 \times 10^{-4} \\
(\mathrm{Ti} \mu \rightarrow \mathrm{Ti} e)^{*)}\end{array}$ & \\
\hline$\left(\bar{d} \gamma^{\rho} P d\right)\left(\bar{\nu}_{\mu} \gamma_{\rho} L \nu_{e}\right)$ & $\begin{array}{c}\left|\varepsilon_{\mu e}^{d P}\right|<7.7 \times 10^{-4} \\
(\mathrm{Ti} \mu \rightarrow \mathrm{Ti} e)^{*)}\end{array}$ & \\
\hline$\left(\bar{e} \gamma^{\rho} P e\right)\left(\bar{\nu}_{\tau} \gamma_{\rho} L \nu_{e}\right)$ & $\begin{array}{c}\left|\varepsilon_{\tau e}^{e P}\right|<2.9 \\
(\tau \rightarrow e \bar{e} e)^{*)} \\
\left|\varepsilon_{\tau e}^{e L}\right|<0.4,\left|\varepsilon_{\tau e}^{e R}\right|<0.7 \\
\text { LSND }\end{array}$ & $\begin{array}{c}\left|\varepsilon_{\tau e}^{e L}\right|<0.02,\left|\varepsilon_{\tau e}^{e R}\right|<0.04 \\
\text { leptonic } s_{W}^{2} \text { at nufact }\end{array}$ \\
\hline$\left(\bar{u} \gamma^{\rho} P u\right)\left(\bar{\nu}_{\tau} \gamma_{\rho} L \nu_{e}\right)$ & $\begin{array}{c}\left|\varepsilon_{\tau e}^{u P}\right|<1.6 \\
(\tau \rightarrow e \rho)^{*)} \\
\left|\varepsilon_{\tau e}^{u P}\right|<0.5 \\
\text { CHARM }\end{array}$ & $\begin{array}{c}\left|\varepsilon_{\tau e}^{u P}\right|<0.03 \\
s_{W}^{2} \text { in DIS at nufact }\end{array}$ \\
\hline$\left(\bar{d} \gamma^{\rho} P d\right)\left(\bar{\nu}_{\tau} \gamma_{\rho} L \nu_{e}\right)$ & $\begin{array}{c}\left|\varepsilon_{\tau e}^{d P}\right|<1.6 \\
\left.(\tau \rightarrow e \rho)^{*}\right) \\
\left|\varepsilon_{\tau e}^{d P}\right|<0.5 \\
\text { CHARM }\end{array}$ & $\begin{array}{c}\left|\varepsilon_{\tau e}^{d P}\right|<0.03 \\
s_{W}^{2} \text { in DIS at nufact }\end{array}$ \\
\hline
\end{tabular}




\section{References}

[1] T. Toshito [Super-Kamiokande Collaboration], arXiv:hep-ex/0105023.

[2] Q. R. Ahmad et al. [SNO Collaboration], Phys. Rev. Lett. 89 (2002) 011301 [arXiv:nucl-ex/0204008].

[3] P. Lipari and M. Lusignoli, Phys. Rev. D60 (1999) 013003 [arXiv:hep-ph/9901350].

[4] G. L. Fogli, E. Lisi, A. Marrone, G. Scioscia, Phys. Rev. D60 (1999) 053006 [arXiv: hep-ph/9904248].

[5] N. Fornengo, M. Maltoni, R. Tomas Bayo and J.W.F. Valle, Phys. Rev. D65 (2002) 013010 [arXiv:hep$\mathrm{ph} / 0108043]$.

[6] [KamLAND Collaboration], arXiv:hep-ex/0212021.

[7] L. Wolfenstein, Phys. Rev. D17 (1978) 2369; S.P. Mikheev and A.Yu. Smirnov, Yad. Fiz. 42 (1985) 1441 [Sov. J. Nucl. Phys. 42 (1985) 913]; F.J. Botella, C.S. Lim, and W.J. Marciano, Phys. Rev. D35 (1987) 896; J.W.F. Valle, Phys. Lett. B199 (1987) 432; E. Roulet, Phys. Rev. D44 (1991) 935; M.M. Guzzo, M. Masiero, and S.T. Petcov, Phys. Lett. B260 (1991) 154.

[8] S. Bergmann, M. M. Guzzo, P. C. de Holanda, P. I. Krastev and H. Nunokawa, Phys. Rev. D62 (2000) 073001 [arXiv:hep-ph/0004049]; M.M. Guzzo, H. Nunokawa, P. C. de Holanda, O. L. G. Peres, Phys. Rev. D64 (2001) 097301 [arXiv:hep-ph/0012089]; M. Guzzo, P. C. de Holanda, M. Maltoni, H. Nunokawa, M. A. Tortola and J. W. Valle, Nucl. Phys. B629 (2002) 479 [arXiv:hep-ph/0112310].

[9] H. K. Dreiner and G. Moreau, arXiv:hep-ph/0211354.

[10] L. M. Johnson and D. W. McKay, Phys. Rev. D61 (2000) 113007 [arXiv:hep-ph/9909355].

[11] T. Ota and J. Sato, Phys. Lett. B545 (2002) 367 [arXiv:hep-ph/0202145].

[12] A. Datta, R. Gandhi, B. Mukhopadhyaya and P. Mehta, Phys. Rev. D64 (2001) 015011 [arXiv:hep$\mathrm{ph} / 0011375]$.

[13] A. M. Gago, M. M. Guzzo, H. Nunokawa, W. J. Teves and R. Zukanovich Funchal, Phys. Rev. D64 (2001) 073003 [arXiv:hep-ph/0105196].

[14] T. Ota, J. Sato and N. a. Yamashita, Phys. Rev. D65 (2002) 093015 [arXiv:hep-ph/0112329].

[15] P. Huber and J.W.F. Valle, Phys. Lett. B523 (2001) 151 [arXiv:hep-ph/0108193].

[16] P. Huber, T. Schwetz and J. W. Valle, Phys. Rev. Lett. 88 (2002) 101804 [arXiv:hep-ph/0111224]; P. Huber, T. Schwetz and J. W. Valle, Phys. Rev. D66 (2002) 013006 [arXiv:hep-ph/0202048].

[17] M. Campanelli and A. Romanino, Phys. Rev. D66 (2002) 113001 [arXiv:hep-ph/0207350].

[18] Z. Berezhiani and A. Rossi, Phys. Lett. B535 (2002) 207 [arXiv:hep-ph/0111137].

[19] V.D. Barger, R.J.N. Phillips and K. Whisnant, Phys. Rev. D44 (1991) 1629.

[20] Y. Grossman, Phys. Lett. B359 (1995) 141 [arXiv:hep-ph/9507344].

[21] M. C. Gonzalez-Garcia, Y. Grossman, A. Gusso and Y. Nir, Phys. Rev. D64 (2001) 096006 [arXiv:hep$\mathrm{ph} / 0105159]$.

[22] S. Bergmann, H. V. Klapdor-Kleingrothaus and H. Pas, Phys. Rev. D62 (2000) 113002 [arXiv:hepph/0004048]; K. S. Babu and C. N. Leung, Nucl. Phys. B619 (2001) 667 [arXiv:hep-ph/0106054].

[23] S. Bergmann, Y. Grossman and D. M. Pierce, Phys. Rev. D61 (2000) 053005 [arXiv:hep-ph/9909390].

[24] see e.g. G. Raffelt, "Stars as Laboratories for Fundamental Physics: The Astrophysics of Neutrinos, Axions, and Other Weakly Interacting Particles", University of Chicago Press (1996).

[25] S. Mansour and T. K. Kuo, Phys. Rev. D58 (1998) 013012 [arXiv:hep-ph/9711424]; S. Bergmann and A. Kagan, Nucl. Phys. B538 (1999) 368 [arXiv:hep-ph/9803305].

[26] G. L. Fogli, E. Lisi, A. Mirizzi and D. Montanino, Phys. Rev. D66 (2002) 013009 [arXiv:hep-ph/0202269].

[27] LSND Collaboration, L.B. Auerbach et al., Phys. Rev. D63 (2001) 112001.

[28] CHARM Collaboration, J. Dorenbosch et al. Phys. Lett. B180 (1986) 303. 
[29] CHARM II Collaboration, P. Vilain et al., Phys. Lett. B335 (1994) 246.

[30] NuTeV Collaboration, G.P. Zeller et al., Phys. Rev. Lett. 88 (2002) 091802 [arXiv:hep-ex/0110059].

[31] see, e.g. S. Davidson, S. Forte, P. Gambino, N. Rius and A. Strumia, JHEP 0202 (2002) 037 [arXiv:hep$\mathrm{ph} / 0112302]$.

[32] F. Cuypers and S. Davidson, Eur. Phys. J. C2 (1998) 503 [arXiv:hep-ph/9609487].

[33] M. Maltoni, Nucl. Phys. Proc. Suppl. 114 (2003) 191 [arXiv:hep-ph/0210111].

[34] M. S. Bilenky and A. Santamaria, Nucl. Phys. B420 (1994) 47 [arXiv:hep-ph/9310302].

[35] A. de Gouvea, S. Lola and K. Tobe, Phys. Rev. D63 (2001) 035004 [arXiv:hep-ph/0008085].

[36] A. Pich, Nucl. Phys. Proc. Suppl. 98 (2001) 385 [arXiv:hep-ph/0012297].

[37] J. N. Bahcall, Phys. Rev. Lett. 12 (1964) 300; R. Davies, Phys. Rev. Lett. 12 (1964) 303.

[38] Z. Berezhiani, R. S. Raghavan and A. Rossi, Nucl. Phys. B638 (2002) 62 [arXiv:hep-ph/0111138].

[39] J. N. Bahcall, K. Kubodera and S. Nozawa, Phys. Rev. D 38, 1030 (1988).

[40] J. Bernabeu, T. E. Ericson, E. Hernandez and J. Ros, Nucl. Phys. B378 (1992) 131.

[41] S. Ando, Y. H. Song, T. S. Park, H. W. Fearing and K. Kubodera, Phys. Lett. B555 (2003) 49 [arXiv:nuclth/0206001].

[42] F. Beacom and S. J. Parke, Phys. Rev. D64 (2001) 091302 [arXiv:hep-ph/0106128].

[43] J. W. Chen, K. M. Heeger and R. G. Robertson, arXiv:nucl-th/0210073.

[44] H. H. Chen, Phys. Rev. Lett. 55 (1985) 1534.

[45] J. N. Bahcall, M. C. Gonzalez-Garcia and C. Pena-Garay, Phys. Rev. C66 (2002) 035802 [arXiv:hep$\mathrm{ph} / 0204194]$.

[46] J. Boger et al. [SNO Collaboration], Nucl. Instrum. Meth. A449 (2000) 172.

[47] S. Fukuda et al. [Super-Kamiokande Collaboration], Phys. Lett. B539 (2002) 179 [arXiv:hep-ex/0205075].

[48] Q. R. Ahmad et al. [SNO Collaboration], Phys. Rev. Lett. 89 (2002) 011302 [arXiv:nucl-ex/0204009].

[49] V. D. Barger, A. M. Gago, D. Marfatia, W. J. Teves, B. P. Wood and R. Zukanovich Funchal, Phys. Rev. D65 (2002) 053016 [arXiv:hep-ph/0110393].

[50] J. N. Bahcall, M. H. Pinsonneault and S. Basu, Astrophys. J. 555 (2001) 990 [arXiv:astro-ph/0010346].

[51] A. M. Dziewonski and D. L. Anderson, Phys. Earth Planet. Interiors 25, 297.

[52] J. N. Bahcall, M. Kamionkowski and A. Sirlin, Phys. Rev. D51 (1995) 6146 [arXiv:astro-ph/9502003].

[53] M. L. Mangano et al., arXiv:hep-ph/0105155.

[54] M. Freund, P. Huber and M. Lindner, Nucl. Phys. B615 (2001) 331 [arXiv:hep-ph/0105071].

[55] A. Bueno, M. Campanelli, M. Laveder, J. Rico and A. Rubbia, JHEP 0106 (2001) 032 [arXiv:hep$\mathrm{ph} / 0010308]$.

[56] We thank Paolo Gambino for a discussion of this point. From the table one can see that the estimated nufactory sensitivity is only a factor of a few better than $\mathrm{NuTeV}$ limits, despite aims to make the nufactory near detector more sophisticated than NuTeV's iron scintillator sandwich.

\section{Erratum to Present and future bounds on non-standard neutrino interactions}

The renormalisation group mixing between operators that induce Non-Standard neutrino Interactions, such as $\left(\bar{\nu}_{\tau} \gamma^{\rho} P_{L} \nu_{\mu}\right)\left(\bar{e} \gamma_{\rho} e\right)$, and operators that induce flavour changing rare charged lepton decays(such as $\left.\tau \rightarrow \mu \bar{e} e\right)$, is not present at leading log. This is contrary to the claims of Section 3.2 and we thank Mattias Blennow, Carla Biggio and Enrique Fernandez-Martinez [1] for drawing our attention to this error.

The bounds on non-standard neutrino Interactions (NSI) are affected as follows: there are finite contributions of the same order ${ }^{\ddagger}$, but unlike the leading log, the corrections are renormalisation scheme-dependent. The scheme dependence can be cancelled by matching corrections at the high scale, which are model dependent. Model and scheme independent bounds could be obtained by performing a NLO calculation.

\footnotetext{
${ }^{\ddagger}$ in dimensional regularisation, and unitary gauge. There are additional diagrams in other gauges, but we obtain the same finite part.
} 


\section{Details}

Recall that the NSI operators in which we are interested are of dimension 8 , are $S U(3) \times S U(2) \times U(1)$ invariant, and are constructed out of four fermion and two Higgs fields. A list of the relevant dimension 8 operators can be found in [2]. The operators are constructed such that when the Higgs gets a vev, they induce the vertex

$$
\left(\bar{f} \gamma^{\rho} f\right)\left(\bar{\nu}_{\alpha} \gamma_{\rho} \nu_{\beta}\right)
$$

but not

$$
\left(\bar{f} \gamma^{\rho} f\right)\left(\bar{e}_{\alpha} \gamma_{\rho} e_{\beta}\right) \quad, \quad\left(\bar{f} \gamma^{\rho} f^{\prime}\right)\left(\bar{\nu}_{\alpha} \gamma_{\rho} e_{\beta}\right)
$$

where $f$ is one of $u, d$ or $e_{R}$, and $\alpha, \beta$ are flavour indices. To simplify our calculation, we neglect the possibility that $f=e_{L}$, which implies that $W$ bosons can not be exchanged between the currents.

We want to obtain the contribution to charged lepton vertices such as Eq. 100, from the the combination of electroweak interactions and the New Physics that induces NSI. We opt to approach this problem from a "hard core" Effective Field Theory approach. We imagine the calculation in three steps [3]:

1. At the mass scale $\Lambda_{N S I}$ of the New Physics inducing NSI, match the full theory onto the Standard Model (SM) plus effective operators invariant under SM gauge symmetries. This determines the (modeldependent) dimensionless coefficients $\left\{C_{i}\right\}$ of the effective dimension 8 operators.

We assume that the coefficients of operators which induce the undesirable vertices (100) are found to be zero to all orders in the new interactions that generate them. This assumption gives the weakest bounds on NSI. It needs a significant amount of fine tuning to be arranged; see the discussion in Ref. [1]. It could in principle arise due to a symmetry.

2. Run this effective theory to $m_{W}$.

3. Match at $m_{W}$ onto the $S U(3) \times U(1)$ theory (where there are no $W$ s); there can be calculable, finite terms from the matching at $m_{W}$.

The first step we do not consider further. We are interested in the effective theory calculation, which starts at step two, with the boundary conditions we have assumed in step one.

We do not consider quadratic divergences. This is because we work in the Effective Field Theory formalism where one calculates in dimensional regularisation, runs with massless RGEs, and at the mass scale of each particle, matches the higher scale theory onto the lower scale theory (from which the particle has been removed). In this formalism, quadratic divergences appear in the matching, and by assumption in step one above, they vanish.

\subsection{Running and matching in broken $\mathrm{SU}(2)$}

To be concrete, suppose that the fundamental theory generates an operator which gives the vertex (99), for $f=e_{R}$, with coefficient $C_{N S I}$. In unitary gauge, one can obtain the first vertex of eqn (100) by $W$ exchange between the neutrino lines (the diagram on the left of figure 9). It is straightforward to verify, using dimensional regularisation, that this diagram is finite. At one loop there is no log, in the lepton massless limit, so no mixing during Renormalisation Group running (step two above) of the neutrino vertex of eqn (99) into the charged lepton vertex of eqn (100). In our original paper, we incorrectly guessed that this anomalous dimension was non-zero so the log was present. However, the diagram has a finite part: at one-loop matching at $m_{W}$, the first vertex Eq. 100 is induced with coefficient

$$
-\frac{3 m_{W}^{2}}{32 \pi^{2}} \frac{C_{N S I}}{\Lambda_{N S I}^{4}} .
$$

Unfortunately, this finite part is not scheme-independent. This problem is discussed in Section 1.3.

We use Naive Dimensional Regularisation for $\gamma_{5}$. We have checked that we get the same result calculating the complete set of diagrams of Fig. 9 in Landau gauge, and in a general $R-\xi$ gauge (where the gauge dependence vanishes as it should).

\subsection{Running and matching in unbroken $\mathrm{SU}(2)$}

In our EFT formalism, where particles are massless when present in the theory, we could imagine SU(2) to be unbroken between $\Lambda_{N S I}$ and $m_{W}$. This perspective allows a simple, SU(2) invariant reformulation of the previous calculation. Consider the operator

$$
\left(\bar{f} \gamma^{\rho} f\right) J_{\rho}^{N S I}
$$



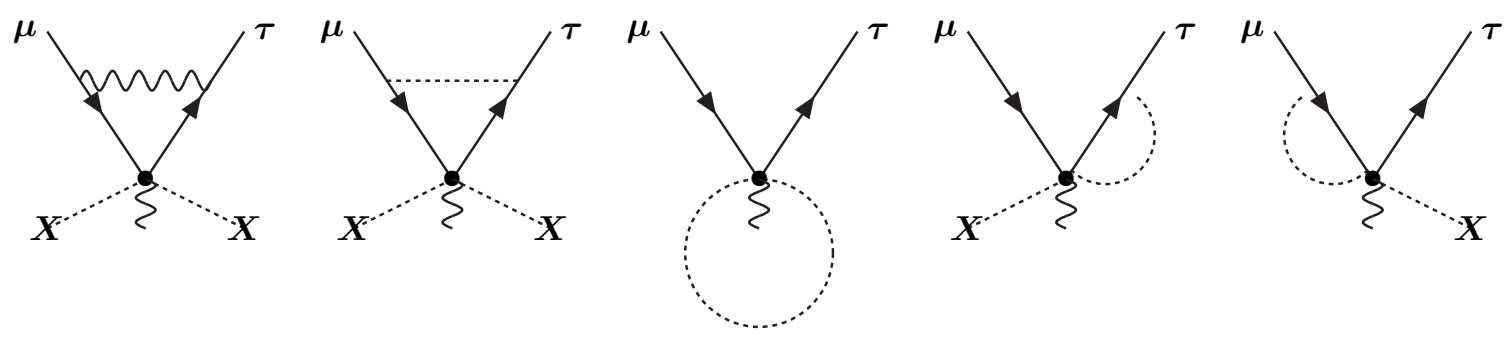

Figure 9: Relevant diagrams for the broken SU(2) calculation.

with

$$
J_{\rho}^{N S I}=\left(\bar{\ell}_{\alpha} H\right) \gamma_{\rho}\left(H^{\dagger} \ell_{\beta}\right) .
$$

Recall $\alpha, \beta$ are flavour indices, $H$ is the Higgs doublet, $\ell_{\alpha}$ are the lepton doublets and we use the diagonal $\mathrm{SU}(2)$ contraction

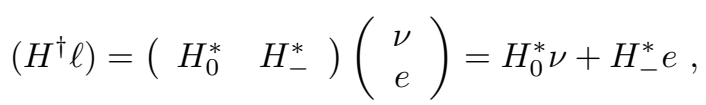

where $\nu$ and $e$ are lefthanded fields.

Interactions among four charged leptons will be generated by Eq. 102 but with $J_{\rho}^{N S I}$ replaced by

$$
J_{\rho}^{C L I}=\left(\bar{\ell}_{\alpha} \tilde{H}\right) \gamma_{\rho}\left(\tilde{H}^{\dagger} \ell_{\beta}\right)
$$

with $\tilde{H}=i \tau_{2} H^{*}$ and $i \tau_{2}$ is the antisymmetric $\mathrm{SU}(2)$ tensor.
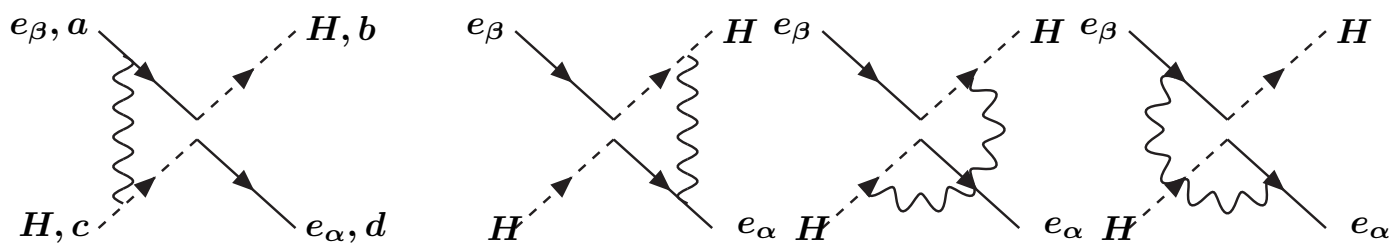

Figure 10: Current mixing diagrams for an unbroken $\mathrm{SU}(2), a, b, c, d$ are $\mathrm{SU}(2)$ indices, same for all diagrams.

We are then interested in the mixing of $J_{\mu}^{N S I}$ into the charged lepton interaction current $J_{\mu}^{C L I}$. We compute the one-loop electroweak corrections to this mixing for an unbroken $\mathrm{SU}(2)$ theory containing gauge bosons and the Higgs doublet. The relevant diagrams are in Fig. 10. We have used dimensional regularization and performed the calculation in a general gauge, checking the cancellation of the dependencies in the gauge parameter. The wave function renormalisation does not contribute to the mixing. We find there are no logarithmic corrections of NSI into CLI, only a finite term which agrees with the result shown in Sec. 1.1 in the theory after SSB.

Alternatively, we can discuss the mixing between triplet and singlet currents

$$
J_{\mu}^{S}=H^{\dagger} H \bar{\ell}_{\alpha} \gamma_{\mu} \ell_{\beta} \quad J_{\mu}^{T}=\sum_{A} H^{\dagger} \tau^{A} H \bar{\ell}_{\alpha} \tau^{A} \gamma_{\mu} \ell_{\beta},
$$

where $\left\{\tau^{A}\right\}$ are the $\mathrm{SU}(2)$ Pauli matrices.

NSI and CLI currents are simply related to singlet and triplet currents by

$$
J_{\mu}^{N S I}=\frac{1}{2} J_{\mu}^{S}+\frac{1}{2} J_{\mu}^{T}, \quad J_{\mu}^{C L I}=\frac{1}{2} J_{\mu}^{S}-\frac{1}{2} J_{\mu}^{T} .
$$

In this case, after inclusion of wave function renormalisation we find that:

- Singlet and triplet currents do not mix at all.

- Singlet and and triplet currents, at one loop, have the same anomalous dimension.

- In naive dimensional regularisation singlet and triplet currents obtain different finite contributions which once translated into the NSI and CLI currents reproduce the finite contribution shown before. 


\subsection{Renormalisation scheme dependence}

If one performs renormalisation group resummation in step two of our EFT prescription, it is well-known [3] that one-loop finite corrections are of the same order (in the $\mathrm{SU}(2)$ coupling $\alpha_{2}$ ) as the two-loop anomalous dimensions. Therefore, to obtain a renormalisation-scheme independent result, we could compute the coefficient of the logarithm arising in the two-loop mixing of $J^{N S I}$ into $J^{S}$. A useful discussion of the twoloop QCD anomalous dimension for a four-quark operator (similar to our four-leg operator, but with scalars replaced by fermions), using various prescriptions for $\gamma_{5}$, can be found in [4].

The SU(2) gauge coupling runs more slowly than $\alpha_{Q C D}$, so one may wonder whether it is neccessary to perform RG resummation. Without it, the two-loop anomalous dimension is $\mathcal{O}\left(\alpha_{2}^{2}\right)$ and can be neglected. The complete calculation at given order in $\alpha_{2}$ should be renormalisation scheme independent, if we do perturbation theory without resummation (notice that we discuss renormalisation scheme dependence, not scale dependence). And, the complete calculation at $\mathcal{O}\left(\alpha_{2}\right)$ includes one loop matching, at the high scale $\Lambda_{N S I}$, of the full theory onto the effective theory. These matching coefficients are clearly model-dependent. So if we do not use the EFT prescription of matching-resummation-matching, then the finite bit we have obtained must be summed with the (model-dependent) finite bits from the high scale matching. Only if we resum, do the scheme-dependent $\mathcal{O}\left(\alpha_{2}\left(m_{W}\right)\right)$ terms cancel between the finite part and the anomalous dimension. This would allow a result which is formally independent of the high-scale theory.

\section{References}

[1] M. Blennow, C. Biggio and E. Fernandez-Martinez, arXiv:0902.0607.

[2] Z. Berezhiani and A. Rossi, Phys. Lett. B 535 (2002) 207 [arXiv:hep-ph/0111137].

[3] A. J. Buras, Les Houches 1997, Probing the standard model of particle interactions [arXiv:hep$\mathrm{ph} / 9806471]$.

[4] A. J. Buras and P. H. Weisz, Nucl. Phys. B 333 (1990) 66. 